\title{
Consistency of the dynamical high-scale type-I seesaw mechanism
}

\author{
Sanjoy Mandalø, ${ }^{1, *}$ Rahul Srivastava, ${ }^{2, \dagger}$ and José W. F. Valle ${ }^{1, \$}$ \\ ${ }^{1}$ AHEP Group, Institut de Física Corpuscular-CSIC/Universitat de València, \\ Parc Científic de Paterna. C/ Catedrático José Beltrán, 2 E-46980 Paterna (Valencia), Spain \\ ${ }^{2}$ Department of Physics, Indian Institute of Science Education and Research-Bhopal, \\ Bhopal Bypass Road, Bhauri, Bhopal 462066, India
}

(Received 6 March 2020; revised manuscript received 1 June 2020; accepted 5 June 2020; published 22 June 2020)

\begin{abstract}
We analyze the consistency of electroweak breaking within the simplest high-scale $\mathrm{SU}(3)_{\mathrm{c}} \otimes \mathrm{SU}(2)_{\mathrm{L}} \otimes$ $\mathrm{U}(1)_{\mathrm{Y}}$ type-I seesaw mechanism. We derive the full two-loop renormalization group equations of the relevant parameters, including the quartic Higgs self-coupling of the Standard Model. For the simplest case of bare "right-handed" neutrino mass terms we find that, with large Yukawa couplings, the Higgs quartic self-coupling becomes negative much below the seesaw scale, so that the model may be inconsistent even as an effective theory. We show, however, that the "dynamical" type-I high-scale seesaw with spontaneous lepton number violation has better stability properties.
\end{abstract}

DOI: $10.1103 /$ PhysRevD.101.115030

\section{INTRODUCTION}

The discovery of a scalar particle with $125 \mathrm{GeV}$ mass plays a central role within particle physics [1,2]. In particular, the precise Higgs boson mass measurement determines the value of the quartic coupling in the scalar potential at the electroweak scale and allows one to study its behavior all the way up to high energies. Given the measured values of Standard Model parameters such as the top quark and Higgs boson masses, we know that the Higgs quartic coupling remains perturbative after renormalization group equations (RGEs) are used to evolve it to high energies. However, the stability of the fundamental vacuum may fail at mass scales below the fundamental Planck scale [3].

Another most important milestone in particle physics has been the discovery of neutrino oscillations [4,5]. This implies the existence of neutrino masses [6] and hence new physics that can produce them [7]. Electroweak vacuum stability can be substantially affected in the presence of a dynamical seesaw mechanism $[8,9] .{ }^{1}$

Here we examine more closely the issue of the consistency of the Higgs vacuum within type-I seesaw extensions

\footnotetext{
*smandal@ific.uv.es

†rahul@iiserb.ac.in

*valle@ific.uv.es

${ }^{1}$ Here we will focus on stability within high-scale seesaw. For discussions of low-scale seesaw see $[10,11]$.

Published by the American Physical Society under the terms of the Creative Commons Attribution 4.0 International license. Further distribution of this work must maintain attribution to the author(s) and the published article's title, journal citation, and DOI. Funded by SCOAP.
}

of the $\mathrm{SU}(3)_{\mathrm{c}} \otimes \mathrm{SU}(2)_{\mathrm{L}} \otimes \mathrm{U}(1)_{\mathrm{Y}}$ Standard Model with an ungauged lepton number [12]. For "sizeable" Yukawa coupling, $Y_{\nu} \sim \mathcal{O}(1)$, in order to reproduce the required neutrino masses, heavy neutrinos must lie at mass scale $M_{N} \sim \mathcal{O}\left(10^{14} \mathrm{GeV}\right)$. This characterizes the case of genuine "high-scale" type-I seesaw constructions. We stress that $\mathrm{SU}(3)_{\mathrm{c}} \otimes \mathrm{SU}(2)_{\mathrm{L}} \otimes \mathrm{U}(1)_{\mathrm{Y}}$ seesaw extensions can be formulated with any number of "right-handed" neutrinos, since they carry no anomaly. Here for definiteness, we start from the minimalistic $(3,1)$ model containing only one righthanded neutrino, in addition to the 3 known left-handed neutrinos [12]. We start from this "missing partner" seesaw, aware that, by itself, it does not provide a fully realistic picture, since only one neutrino mass scale arises at the tree level [12]. However, there are interesting and realistic variants where the "missing partner" seesaw scale can be identified with the "atmospheric scale", while the "solar" mass scale is generated by radiative corrections that could arise, for example, from a "dark matter" sector [13]. We therefore take such "missing partner" seesaw as our reference scheme.

We show that, although it has better stability properties than the fully sequential $(3,3)$ seesaw mechanism, for sizeable magnitudes of the Yukawa couplings the Higgs potential of the minimal $(3,1)$ seesaw becomes unstable even below the seesaw scale. The situation can only get worse by having more right-handed neutrinos, in the $(3,2)$ seesaw or in the "sequential" $(3,3)$ seesaw. In this sense, too, it makes sense to take such a "missing partner" $(3,1)$ seesaw as the reference scheme. An important implication of this missing partner type-I seesaw is the existence of a lower bound on the neutrinoless double beta decay rate even for normal ordered neutrino masses [14]. 
We then show explicitly that vacuum stability can be improved naturally if one implements spontaneous violation of lepton number. This is characterized by the existence of a physical Nambu-Goldstone boson, dubbed majoron $[15,16]$. We show how the extra scalars required to implement spontaneous lepton number violation play a key role to improve stability properties. Indeed, their couplings can easily restore stability of the electroweak symmetry breaking even if the lepton number violation scale is high, as required to fit neutrino masses in this case. We also analyze the scale at which instability sets in as a function of the magnitude of the Yukawa coupling relevant for generating neutrino mass in $(3,1)$, as well as the conventional $(3,3)$ seesaw case.

This work is organized as follows: In Sec. II we revisit the vacuum stability problem in the Standard Model showing that the Higgs quartic coupling becomes negative when RGEs-evolved to high scales. In Sec. III, we describe the neutrino mass generation in type-I seesaw and type-I seesaw with majoron extensions. We then show in Sec. IV that the vacuum stability problem becomes worse in highscale type-I seesaw Standard Model extensions. We then focus on the majoron extension of the canonical type-I seesaw. We show how the majoron helps stabilize the Higgs vacuum, which can be made stable all the way up to Planck scale. In addition the majoron could provide a viable dark matter candidate [17-21], thereby solving another basic problem in particle physics. Finally, we conclude and summarize our main results in Sec. VIII.

\section{HIGGS VACUUM IN THE STANDARD MODEL}

Let us briefly revisit the status of the electroweak (EW) vacuum within the Standard Model. For a long time the Higgs boson was the "last" missing piece of the theory. The discovery of a scalar particle with mass $m_{H} \approx 125 \mathrm{GeV}$ at the Large Hadron Collider (LHC) is very suggestive that it could be the long-awaited Standard Model Higgs boson. While further work is still needed to unambiguously establish this, current data indicates that its couplings and decay properties are close to the Standard Model Higgs expectations. If, indeed, this is the case, the next question is, given that so far we have not seen any evidence for new particles at the LHC, whether the Standard Model can be the final theory. The answer is obviously no, since the Standard Model predicts neutrinos to be massless and there is no viable Standard Model candidate for cosmological dark matter.

For the moment we put these two issues aside, and ask ourselves whether there are other compelling hints that the Standard Model cannot be the final theory up to Planck scale. Indeed, there are several other theoretical and aesthetical arguments against this being the case. For example, achieving the unification of forces and the improving the hierarchy/fine-tuning/naturalness problem. However, the "Higgs discovery" has facilitated us to study
TABLE I. $\overline{\mathrm{MS}}$ values of the main input parameters at the top quark mass scale, $m_{t}=173 \pm 0.4 \mathrm{GeV}$.

\begin{tabular}{cccccc}
\hline \hline & $g_{1}$ & $g_{2}$ & $g_{3}$ & $y_{t}$ & $\lambda_{\mathrm{SM}}$ \\
\hline$\mu\left(m_{t}\right)$ & 0.462607 & 0.647737 & 1.16541 & 0.93519 & 0.126115 \\
\hline \hline
\end{tabular}

the high energy behavior of the Standard Model. As an example, in this work we address the stability of Higgs vacuum at energies far above the electroweak scale.

The detailed analysis of the Higgs vacuum within the Standard Model has been carried out in [22-27]. For completeness here we revisit this analysis. This serves us to calibrate our Renormalization Group analysis against known results. Although in dedicated Standard Model studies there are some partial 3-loop results [27], to compare the seesaw and Standard Model results it will suffice for us to stay at the two-loop level. In our analysis we adopt the $\overline{\mathrm{MS}}$ scheme, taking the parameter values at low scale as the input values [3]. In particular, the Higgs pole mass is taken as the current best fit value of $m_{H}=125.18 \pm 0.16 \mathrm{GeV}$, the top quark pole mass is taken as $m_{t}=173 \pm 0.4 \mathrm{GeV}$, and the strong coupling constant $\alpha_{s}\left(M_{Z}\right)=0.1184 \pm 0.0007$. Using these experimental values, we adopt the "On-Shell" renormalization scheme in order to express the renormalized parameters directly in terms of the physical observables and then relate the on-shell parameters to the $\overline{\mathrm{MS}}$ parameters in a way similar to [27]. In Table I we list the $\overline{\mathrm{MS}}$ input values of the relevant parameters at the top mass $m_{t}$ scale.

Taking the initial $\overline{\mathrm{MS}}$ values of Table I as input values, we then RGEs-evolve the Standard Model parameters to higher scales as shown in Fig. 1.

Taking into account the updated input parameter values, our two-loop results are in good agreement with earlier ones. Tiny differences arise mainly due to the increased current precision of the experimental numbers. We stress

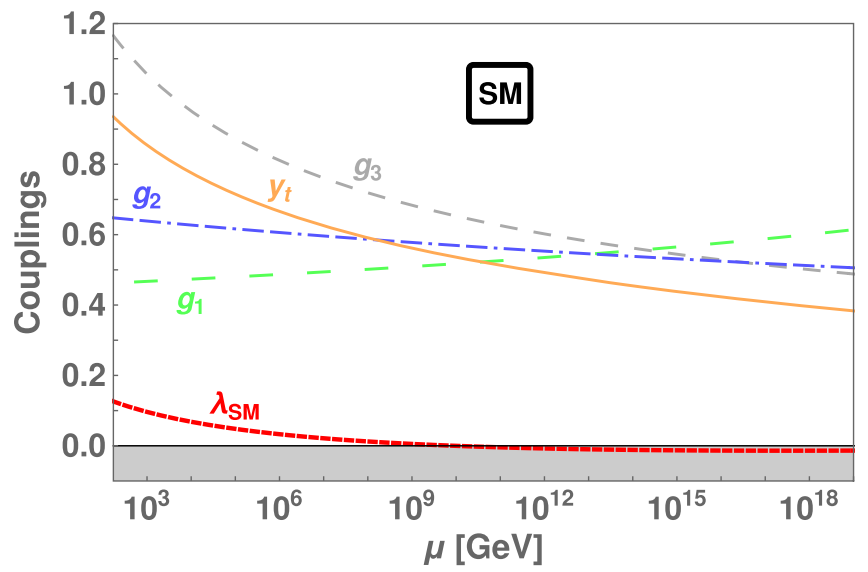

FIG. 1. The RGEs evolution of the Standard Model gauge couplings $g_{1}, g_{2}, g_{3}$, the top quark Yukawa coupling $y_{t}$, and the quartic Higgs boson self-coupling $\lambda_{\mathrm{SM}}$. 
that an in-depth reanalysis of the Standard Model Higgs is not the goal of our paper, but rather the comparison of Standard Model and seesaw scenarios. Hence, we refrain from performing a sensitivity analysis of Higgs vacuum stability and its dependence on the input parameter errors. Indeed, in the seesaw scenarios of interest to us, such tiny effects are negligible when compared to the effects of the new Yukawa couplings.

Notice from Fig. 1 that the Standard Model Higgs quartic coupling $\lambda_{\mathrm{SM}}$ becomes negative at $\mu \simeq 10^{10} \mathrm{GeV}$. This would imply that the Higgs potential is unbounded from below and the Higgs vacuum would be unstable. A dedicated analysis shows that, in fact, the Standard Model Higgs vacuum is not unstable, but rather metastable ${ }^{2}$ with a very long lifetime [27].

\section{NEUTRINO MASS GENERATION}

As already mentioned, the Standard Model cannot be the final theory up to the Planck scale, as it has massless neutrinos and no viable candidate for dark matter. Hence the vacuum stability issue must be reconsidered. We now do this adopting simple seesaw extensions of the Standard Model. We show that, above the seesaw scale, the Higgs vacuum stability can be completely dominated by the new couplings. Hence it suffices for our purposes to discuss electroweak vacuum stability at the two-loop level.

\section{A. Dimension-five operator}

Within the Standard Model neutrinos are massless. However, as first noted by Weinberg [28], nonzero masses will arise from an effective nonrenormalizable dimensionfive operator characterizing lepton number nonconservation. The effective Lagrangian reads

$$
-\mathcal{L}_{\nu}^{d=5}=\frac{1}{2}\left(\bar{\ell}_{L} H\right) . \kappa \cdot\left(H^{T} \ell_{L}^{c}\right)+\text { H.c. },
$$

where $\kappa$ is the $3 \times 3$ symmetric coupling matrix with negative mass dimension, and, for brevity, we have suppressed the generation indices. When the electroweak symmetry breaking occurs, the Higgs gets vacuum expectation value (vev) $\langle H\rangle=\frac{v}{\sqrt{2}}$ with $v=246 \mathrm{GeV}, H$ being the Standard Model Higgs doublet. This leads to a Majorana mass matrix for the left-handed neutrinos given as

$$
m_{\nu} \equiv \kappa \frac{v^{2}}{2}
$$

which leads to light neutrino masses and lepton number violation by two units. There are many ways to generate $\kappa$

\footnotetext{
${ }^{2}$ Standard Model vacuum stability is sensitive to input parameter values, in particular the top-quark mass.
}

as a result of postulating new mediator particles. A very simple "UV-completion" is the type-I seesaw mechanism.

\section{B. Type-I seesaw mechanism}

The most general "type-I seesaw" mechanism is the one formulated in terms of just the $\mathrm{SU}(3)_{c} \otimes \mathrm{SU}(2)_{\mathrm{L}} \otimes \mathrm{U}(1)_{\mathrm{Y}}$ structure characterizing the Standard Model, without extra gauge symmetry [12]. One postulates the existence of gauge singlet "right-handed neutrinos", $\nu_{R_{i}}, i=1,2, \ldots n$, whose mass term is obviously gauge invariant. Neutrino masses arise from the exchange of "right-handed neutrino" mediators whose multiplicity is arbitrary since, as gauge singlets, they carry no anomaly. The relevant part of the Lagrangian is written as

$$
-\mathcal{L}=\sum_{a, i} Y_{\nu}^{a i} \bar{\ell}_{L}^{a} \tilde{H} \nu_{R_{i}}+\frac{1}{2} \sum_{i, j} M_{R}^{i j} \overline{\nu_{R_{i}}^{c}} \nu_{R_{j}}+\text { H.c. }
$$

where $\ell_{L}^{a}=\left(\nu_{L}^{a}, l_{L}^{a}\right)^{T}$ with $a=1,2,3$ denotes the three families of left-handed lepton doublets, while $i, j=$ $1,2, \ldots n$ labels the right-handed singlet neutrinos, and, as before, $H$ is the Standard Model Higgs doublet. After electroweak symmetry breaking the full neutrino mass matrix is expressed as

$$
\mathcal{M}_{\nu}=\left(\begin{array}{cc}
0 & m_{D} \\
m_{D}^{T} & M_{R}
\end{array}\right)
$$

where $m_{D}=\frac{Y_{\nu}}{\sqrt{2}} v$ is the "Dirac mass matrix".

Being $\mathrm{SU}(3)_{\mathrm{c}} \otimes \mathrm{SU}(2)_{\mathrm{L}} \otimes \mathrm{U}(1)_{\mathrm{Y}}$ invariant, the righthanded neutrino "Majorana mass matrix" $M_{R}^{i j}$ entries can be much larger than the EW scale, $\left|M_{R}^{i j}\right| \gg v$, implying $\left|\frac{m_{D}^{a j}}{M_{R}^{i j}}\right| \ll 1$. Hence the mass matrix in Eq. (3) can be blockdiagonalized perturbatively in an exponential series, Eq. (3.1) in [16]. ${ }^{3}$ The two diagonalized blocks correspond to "light" and "heavy" neutrino mass matrices, denoted as $m_{\nu}^{a b}$ and $M_{N}^{a b}$, respectively, which can be written symbolically as:

$$
\begin{aligned}
m_{\nu}^{a b} & =\frac{1}{2}\left[M_{R}^{a b}-\left(\sqrt{M_{R}^{2}+4 m_{D}^{2}}\right)^{a b}\right] \\
M_{N}^{a b} & =\frac{1}{2}\left[M_{R}^{a b}+\left(\sqrt{M_{R}^{2}+4 m_{D}^{2}}\right)^{a b}\right] .
\end{aligned}
$$

To leading order the mass matrix elements for light neutrinos $m_{\nu}^{a b}$ and heavy neutrinos $M_{N}^{a b}$ are given as

$$
m_{\nu}^{a b} \simeq-m_{D}^{a i}\left(M_{R}^{-1}\right)^{i j}\left(m_{D}^{T}\right)^{j b}+\text { higher order terms, }
$$

$M_{N}^{a b} \simeq M_{R}^{a b}+m_{D}^{a i}\left(M_{R}^{-1}\right)^{i j}\left(m_{D}^{T}\right)^{j b}+$ higher order terms,

\footnotetext{
${ }^{3}$ We are now using the series expansion in Ref. [16] for the case of explicit lepton number violation.
} 
where the negative sign in (5) can be absorbed ${ }^{4}$ through field redefinition. The full expression for the diagonalizing matrix is found in [16], as Eq. (3.5). The light neutrino mass matrix in (5) is further diagonalized by a unitary matrix $U_{\nu}$ in the light neutrino sector $\nu^{a} ; a=1,2,3$. This famous type-I seesaw formula links the smallness of the light neutrino masses to the heaviness of the right-handed neutrinos $\nu_{R}$.

\section{The missing partner type-I seesaw mechanism}

As already mentioned, since $\nu_{R}$ 's are Standard Model gauge singlets, their number $n$ need not match the number of left-handed ones. Depending on the value of " $n$ " many possibilities can be envisaged. Here we consider the case $n \leq 3$ of "high-scale" constructions. 5

The observation of neutrino oscillations [4,5] proves that two of the three "active" neutrinos are massive [6]. However, there is so far no indication for a finite mass for the lightest neutrino. Indeed, the Katrin experiment has derived an upper limit of $1.1 \mathrm{eV}$ (at 90\% C.L.) on the absolute mass scale of neutrinos [34] from the Tritium endpoint spectrum. This bound applies irrespective of whether neutrinos are Dirac or Majorana particles. On the other hand, cosmological observations indicate that $\sum m_{a} \leq 0.12 \mathrm{eV}[35,36]$. Hence, for "sizeable" Yukawa coupling values, $Y_{\nu} \sim \mathcal{O}(1)$, this bound is satisfied for heavy neutrino masses $M_{N} \sim \mathcal{O}\left(10^{14} \mathrm{GeV}\right)$.

In order to account for the current oscillation evidence for neutrino mass it suffices to have a "missing partner" seesaw mechanism with $n=2$, since in this case both solar and atmospheric scales can be produced by the seesaw. Following the general formulation in [12] we call such a scheme in which one left-neutrino has no right partner, $(3,2)$ seesaw. In this case each right-handed neutrino mediates the generation of the corresponding scale, solar, or atmospheric.

Notice, however, that the minimal type-I seesaw mechanism is the one in which only one right-handed neutrino is added to the Standard Model. This $(3,1)$ scheme is the minimal "missing partner" seesaw, in which two leftneutrinos lack a right-partner and remain massless. It can be viable as part of a bigger scheme in which, for example, the solar scale arises radiatively, hence accounting for the small solar/atmospheric scale ratio. Such scheme is easily obtained by "cloning" the seesaw with some other sector associated, for example, with dark matter. An interesting realization is the scotoseesaw, in which the

\footnotetext{
${ }^{4}$ Note that, even though this negative sign in (5) is physical, related to the $C P$ properties of the light neutrinos, for our purposes they will not be relevant.

${ }^{5}$ Here we discard cases with $n>3$ since having extra fermions can only worsen stability. An interesting example would be the $(3,6)$ seesaw scheme, which includes the template for the sequential "low-scale" seesaw, including both the inverse seesaw $[29,30]$ and the linear seesaw mechanisms [31-33].
}

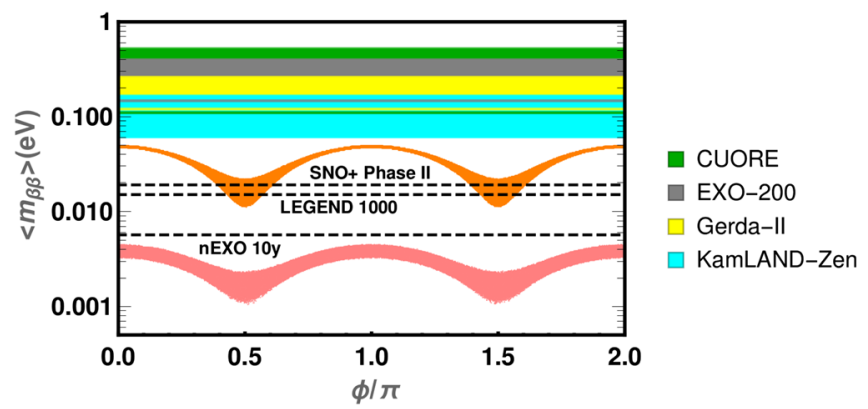

FIG. 2. Amplitude for neutrinoless double beta decay in missing partner seesaw. The horizontal bands represent the limits from current experiments, while the horizontal dashed lines show the maximum reach of future experiments (see text).

atmospheric scale is seesaw-induced, while the solar scale has scotogenic origin [13].

An implication of the missing partner seesaw schemes is a prediction for the parameter $m_{\beta \beta}$ describing the amplitude for neutrinoless double beta decay versus the (relative) massive neutrino Majorana phase, shown in Fig. 2.

The lower band corresponds to normal mass ordering and the upper one to inverted. Their narrow widths reflect the small allowed spread in neutrino oscillation parameters [6]. Notice that, in contrast to the general "complete" $(3,3)$ seesaw, in the missing partner there can be no cancellation, so that nonzero neutrinoless double beta decay is predicted, even if neutrinos are normal-ordered. The horizontal bands in Fig. 2 show the reach of present experiments: CUORE (green, limits: 0.11-0.52 eV) [37], EXO-200 (grey, limits: $0.147-0.398 \mathrm{eV}$ ) [38], Gerda-II (yellow, limits: 0.120 $0.260 \mathrm{eV}$ ) [39] and KamLAND-Zen (cyan, limits: 0.061$0.165 \mathrm{eV}$ ) [40]. The horizontal lines indicate the maximum estimated experimental sensitivities ${ }^{6}$ of upcoming experiments: SNO+ Phase-II $(0.019 \mathrm{eV})$ [41], LEGEND - 1000 (0.015) [42] and nEXO - $10 \mathrm{yr}(0.0057)$ [43]. One sees from Fig. 2 that, although the upcoming experiments are only sensitive to inverted ordering, the detectability chances improve in the "missing partner" as compared to the expectations of a generic "complete" seesaw mechanism. This brings hope that upcoming experiments may be able to measure, for the first time, the relevant Majorana phase.

To sum up, in what follows we take the missing partner seesaw as our reference benchmark, because of its minimality and notational simplicity, and also because of the fact that having extra fermions can only worsen stability of the Higgs potential. In addition, in the "complete" seesaw picture one looses the neutrinoless double beta decay prediction in Fig. 2.

However, in Sec. VII we explicitly compare our results with those obtained for the sequential $(3,3)$ seesaw. Moreover, all the relevant renormalization group equations

\footnotetext{
${ }^{6}$ Here we made the most optimistic assumptions concerning nuclear matrix element uncertainties.
} 


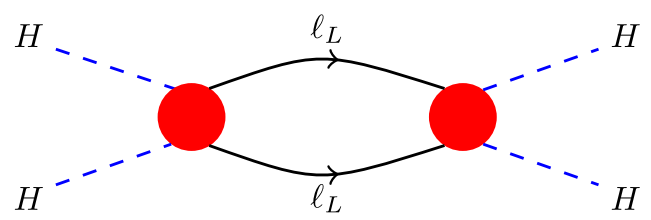

FIG. 3. Effect of Weinberg's effective operator on the Higgs quartic interaction in the effective theory.

given in the Appendix assume the conventional $(3,3)$ seesaw picture.

\section{HIGGS VACUUM STABILITY AND NEUTRINO MASS}

We saw how the Standard Model vacuum is not absolutely stable. Instead, with the present measured values of Higgs and top masses, it is metastable; as the quartic coupling $\lambda_{\mathrm{SM}}$ becomes negative around $\Lambda_{I}^{\mathrm{SM}} \sim 10^{10} \mathrm{GeV}$, the Standard Model instability scale. Before discussing Higgs vacuum stability within type-I seesaw embeddings of the Standard Model we first consider the effective Weinberg operator.

\section{A. Dimension-five operator}

We now discuss the running of the quartic scalar coupling characterizing the EW symmetry breaking sector of the Standard Model in the context of a dimension-five operator picture that results effectively at low-energies from a UV-complete type-I seesaw. Below the scale $\mu=M_{R}$ we integrate out the heavy neutrino $\nu_{R}$, so that the theory is the Standard Model plus an effective dimension-five Weinberg operator $\mathcal{L}_{\nu}^{d=5}$ (with $\kappa=Y_{\nu} M_{R}^{-1} Y_{\nu}^{T}$ ). Below the scale $\mu=M_{R}$, only the Standard Model couplings and $\kappa$ will run. Neglecting the contribution from lepton and light quark Yukawa couplings, the one-loop RGEs are given by [44-46] (see also Ref. [10]),

$$
16 \pi^{2} \beta_{\kappa}=6 y_{t}^{2} \kappa-3 g_{2}^{2} \kappa+\lambda_{\kappa} \kappa
$$

As the Standard Model case, here $y_{t}$ also denotes the top Yukawa coupling and $g_{2}$ is the $S U(2)_{L}$ gauge coupling. We denote the Higgs quartic coupling in this case as $\lambda_{\kappa}$ to distinguish it from the pure Standard Model case. Hence, due to the large top Yukawa coupling $y_{t}$, the coupling $\kappa$ slowly increases with the energy scale $\mu$. As seen in Fig. 3 [10], the same operator which generates the neutrino mass below the scale $\mu=M_{R}$, also provides a correction to the Higgs quartic self-coupling $\lambda_{\kappa}$ below that scale. The contribution from the coupling $\kappa$ on the running of the Higgs quartic coupling $\lambda_{\kappa}$ is of order $v^{2} \kappa^{2}$ and thus negligible [47]. As a result, in the effective theory, the running of $\lambda_{\kappa}$ below the scale $\mu=M_{R}$ will be almost the same as in the Standard Model.

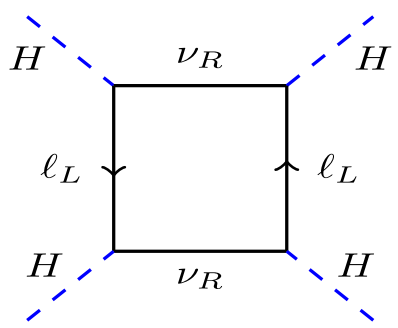

FIG. 4. One-loop correction to the Higgs quartic interaction in the full seesaw theory.

\section{B. Higgs vacuum stability in type-I seesaw}

In what follows, we will be mostly concerned with the effects of sizable Yukawa couplings in the context of high-scale missing partner seesaw and their impact on the stability of the Higgs vacuum. Building upon the discussion of the previous section, we now turn to the region above the scale $\mu=M_{R}$. In this case one has the full theory in which the running of Yukawa coupling $Y_{\nu}$ will have an impact on the running of the Higgs quartic coupling which we now call $\lambda$. This is done so as to distinguish it from both the Standard Model case as well as from the regime where the renormalization group running is performed only with the effective Weinberg operator. Within the type-I seesaw picture, below and above the seesaw scale $\mu=M_{R}$, there will be contributions on Higgs quartic coupling $\lambda$ from the Figs. 3 and 4, respectively. Hence, in order to describe the running of $\lambda$ we need to take into account the matching condition at the scale $\mu=M_{R}$.

For the reasons mentioned in Sec. III B here we focus on the simplest missing partner type-I seesaw mechanism containing a single right-handed neutrino. It provides a clear picture of the impact of seesaw extensions on the Higgs vacuum stability in the simplest possible setting.

As we discussed earlier, below the $\mu=M_{R}$ scale, the theory is an effective Standard Model supplemented by the dimension-five Weinberg operator. However, above the $\mu=M_{R}$ scale the theory is UV-complete, so that all the new couplings in the model like the neutrino Yukawa coupling $Y_{\nu}$ will take part in the system of renormalization group equations and will affect the running of the Standard Model couplings, especially that of $\lambda$.

As a result, the stability of the electroweak vacuum will set a potential limit on how large $Y_{\nu}$ can be. As the new Yukawa coupling $Y_{\nu}$ runs only above the threshold scale $M_{R}$, this can be technically implemented by replacing $Y_{\nu} \rightarrow Y_{\nu} \theta\left(\mu-M_{R}\right)$ in the right hand side of the RGEs of the full theory, given in Appendix B. Here $\theta(x)=1, x>0$ and $\theta(x)=0, x<0$ are the step functions.

Integrating out the heavy neutrinos also introduces threshold corrections to the Standard Model Higgs quartic coupling $\lambda$ [48] at the scale $\mu=M_{R}$. The tree level Higgs potential in the Standard Model is given by 


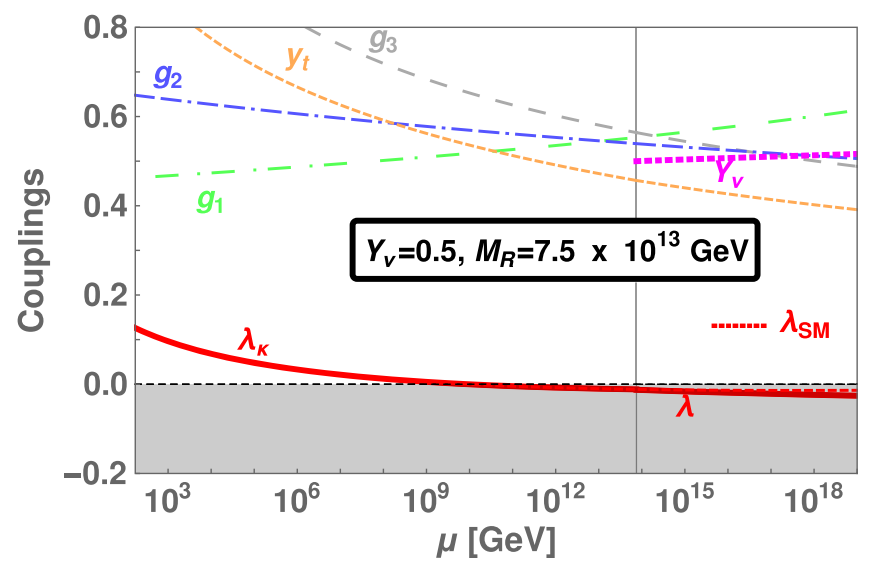

FIG. 5. The continuous (red) curve gives the evolution of the Higgs quartic self-coupling within the minimal $(3,1)$ Type I seesaw scheme. The gauge and Yukawa couplings $g_{1}, g_{2}, g_{3}, y_{t}$, and $Y_{\nu}$ are also indicated by the dashed lines. The light neutrino mass is fixed at $m_{\nu}=0.1 \mathrm{eV}$, corresponding to a heavy neutrino mass $M_{R}$ of $7.5 \times 10^{13} \mathrm{GeV}$. For comparison we show the evolution of the Standard Model coupling $\lambda_{\mathrm{SM}}$, seen as the red dashed line. Finally, $\lambda_{\kappa}$ denotes the Higgs quartic coupling in the effective theory including neutrino mass through the Weinberg operator, while $\lambda$ is the corresponding quartic in the minimal missing-partner Type I seesaw theory.

$$
V\left(H^{\dagger} H\right)=-\mu_{H} H^{\dagger} H+\lambda\left(H^{\dagger} H\right)^{2} .
$$

This will get corrections from higher loop diagrams of Standard Model particles and extra fermion from the type-I seesaw. The one-loop effective potential $V_{1}(h)$ has the form

$$
V_{1}(h)=V_{1}^{\mathrm{SM}}(h)+V_{1}^{\nu}(h),
$$

where $V_{1}^{\mathrm{SM}}(h)$ is the usual one loop Standard Model potential. The one loop potential from the neutrino sector is given by $[48,49]$

$V_{1}^{\nu}(h)=-\frac{1}{32 \pi^{2}}\left(\sum_{i} m_{\nu_{i}}^{4} \log \frac{m_{\nu_{i}}^{2}}{\mu^{2}}+\theta\left(\mu-M_{R}\right) M_{N}^{4} \log \frac{M_{N}^{2}}{\mu^{2}}\right)$.

The matching of the complete and effective theory at threshold requires one to introduce a threshold contribution below $M_{R}, \Delta_{\mathrm{TH}} V=-\frac{1}{32 \pi^{2}}\left(M_{N}^{4} \log \frac{M_{N}^{2}}{M_{R}^{2}}\right)$, whose expansion gives the threshold corrections to the $\mu^{2}$ and $\lambda$ parameters as $\Delta_{\mathrm{TH}} \mu^{2}=\frac{1}{16 \pi^{2}}\left|Y_{\nu}\right|^{2} M_{R}^{2}, \Delta_{\mathrm{TH}} \lambda=-\frac{5}{32 \pi^{2}}\left|Y_{\nu}\right|^{4}$. Hence, we need to consider this shift in $\lambda$ at $\mu=M_{R}$ when solving the RGEs as

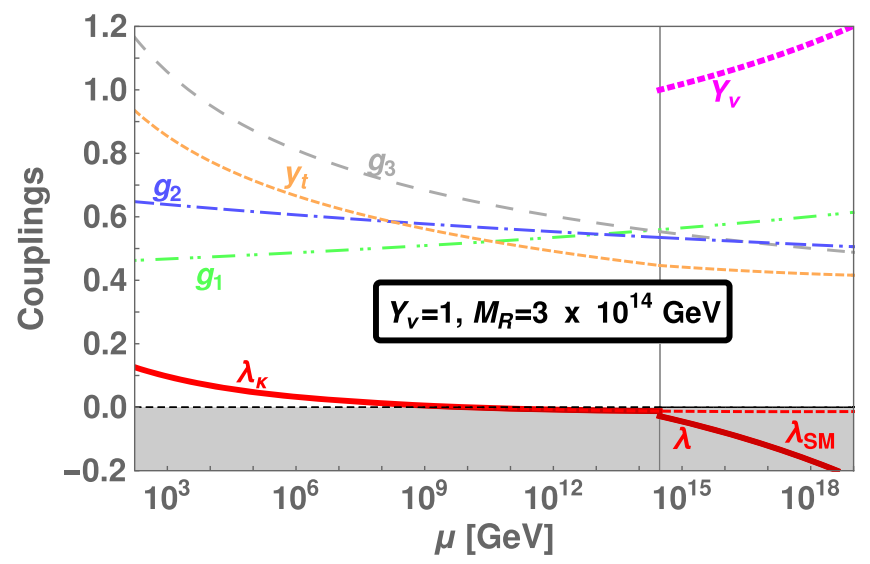

FIG. 6. Same as Fig. 5 but now with $Y_{\nu}=1$ and $M_{R}=$ $3 \times 10^{14}$. In contrast to Fig. $5, \lambda$ shows a marked deviation from $\lambda_{\mathrm{SM}}$ as a result of the larger Yukawa value, $Y_{\nu}=1$. As before, $\lambda_{\mathrm{SM}}$ and $\lambda_{\kappa}$ nearly coincide as the Weinberg operator has negligible impact on RGEs of the quartic Higgs coupling.

$$
\lambda\left(M_{R}\right) \rightarrow \lambda\left(M_{R}\right)-\frac{5}{32 \pi^{2}}\left|Y_{\nu}\right|^{4} .
$$

Having set up our basic scheme, let us start by looking at the impact of the right-handed neutrinos on the stability of the Higgs vacuum. As we discussed at length in Sec. II, the Standard Model RGEs running of the Higgs quartic scalar coupling $\lambda_{\mathrm{SM}}$ are dominated by the top Yukawa, which is the largest coupling present in the theory. As we saw, in this case the Standard Model $\lambda_{\mathrm{SM}}$ coupling becomes negative around the scale $\mu \sim 10^{10} \mathrm{GeV}$. However, within the seesaw completion, above the scale $\mu=M_{R}$ the neutrino Yukawa couplings $Y_{\nu}$ of (2) can completely dominate the RGEs behavior of $\lambda$ as shown in Figs. 5 and 6. Figures 5 and 6 illustrate the effect of the new neutrino Yukawa coupling $Y_{\nu}$ on various other couplings. For illustration we have taken two representative values of Yukawa couplings $Y_{\nu}=0.5$ and 1. One sees how the problem of Higgs vacuum stability becomes more acute in a type-I seesaw completion of the Standard Model. This was expected, since the addition of new fermions tends to destabilize the Higgs vacuum. Notice that, in the regime below the onset of the seesaw mechanism, $\mu \leq M_{R}$, the running of the Higgs quartic coupling $\lambda_{\kappa}$ nearly coincides with $\lambda_{\mathrm{SM}}$. This follows due to the negligible effect of the Weinberg operator on the running of the Higgs quartic coupling. The small negative shift in the $\lambda$ running at the scale $\mu=M_{R}$ results from the matching condition, which becomes clearly visible for larger Yukawa couplings, $Y_{\nu} \sim 1$. Notice that in Figs. 5 and 6 we have chosen a larger seesaw scale, with correspondingly larger "Dirac" neutrino Yukawa coupling values, in order to make the running coupling effects visible in the plots.

For $M_{N} \leq 10^{10} \mathrm{GeV}$, the Yukawa coupling is $Y_{\nu} \leq 10^{-3}$, hence too small to alter the running of $\lambda$ significantly. As a 
result, the vacuum instability problem will persist. However, if regarded as an effective one, the theory remains mathematically self-consistent. For larger $M_{N}$, for example $M_{N}$ close to the unification scale, the type-I seesaw relation (5) implies that $Y_{\nu}$ should also be sizeable. Such a large Yukawa coupling will have a destabilizing effect, worsening the metastability of the Standard Model vacuum. ${ }^{7}$ In fact, now the vacuum can be completely unstable, making the model inconsistent.

In conclusion, in seesaw scenarios the stability properties of the electroweak vacuum will at best be those of the Standard Model Higgs vacuum. In order to enhance Higgs vacuum stability it is desirable to further extend or embed the type-I seesaw [50]. A natural way to do this is to assume spontaneous breaking of lepton number, as we do next.

\section{THE MAJORON COMPLETION}

We now consider the type-I seesaw extensions of the Standard Model, in which lepton number is promoted to a spontaneously broken symmetry within the $\mathrm{SU}(3)_{\mathrm{c}} \otimes$ $\mathrm{SU}(2)_{\mathrm{L}} \otimes \mathrm{U}(1)_{\mathrm{Y}}$ gauge framework $[15,16]$. In addition to the right-handed neutrinos $\nu_{R}$ we add a complex scalar singlet $\sigma$ carrying two units of lepton number. The relevant Lagrangian is given by

$$
\mathcal{L}=-\sum_{a, i} Y_{\nu}^{a i} \bar{\ell}_{L}^{a} \tilde{H} \nu_{R_{i}}-\frac{1}{2} \sum_{i, j} Y_{R}^{i j} \sigma \overline{\nu_{R_{i}}^{c}} \nu_{R_{j}}+\text { H.c. }
$$

The resulting neutrino mass matrices in $\nu_{L}$ and $\nu_{R}$ basis is given by

$$
\mathcal{M}_{\nu}=\left(\begin{array}{cc}
0 & \frac{Y_{\nu} v_{H}}{\sqrt{2}} \\
\frac{Y_{\nu}^{T} v_{H}}{\sqrt{2}} & \frac{Y_{R} v_{\sigma}}{\sqrt{2}}
\end{array}\right) .
$$

The effective light neutrino mass obtained by perturbative diagonalization of the above mass matrix is of the form

$$
m_{\nu} \simeq Y_{\nu} Y_{R}^{-1} Y_{\nu}^{T} \frac{v_{H}^{2}}{\sqrt{2} v_{\sigma}} .
$$

In the presence of the complex scalar singlet $\sigma$, the most general Higgs potential that can drive electroweak and lepton number symmetry breaking is given by [51]

$$
\begin{aligned}
V(\sigma, H)= & -\mu_{H}^{2} H^{\dagger} H-\mu_{\sigma}^{2} \sigma^{\dagger} \sigma+\lambda_{H}\left(H^{\dagger} H\right)^{2} \\
& +\lambda_{\sigma}\left(\sigma^{\dagger} \sigma\right)^{2}+\lambda_{H \sigma}\left(H^{\dagger} H\right)\left(\sigma^{\dagger} \sigma\right) .
\end{aligned}
$$

This potential is bounded from below if $\lambda_{\sigma}, \lambda_{H}$ and $\lambda_{H \sigma}+2 \sqrt{\lambda_{\sigma} \lambda}$ are all positive. In addition to the standard

\footnotetext{
${ }^{7}$ For example, if $M_{N} \sim 10^{14} \mathrm{GeV}$ (which implies $Y_{\nu} \sim 1$ for $m_{\nu} \sim 0.1 \mathrm{eV}$ ), the vacuum lifetime is less than the age of the universe $\tau_{U}$, hence Standard Model metastability is worsened by the effect of this large Yukawa coupling.
}

$\mathrm{SU}(3)_{\mathrm{c}} \otimes \mathrm{SU}(2)_{\mathrm{L}} \otimes \mathrm{U}(1)_{\mathrm{Y}} \quad$ gauge invariance, in the unbroken phase, the theory is also invariant under lepton number. The above potential can develop a minimum for nonzero vacuum expectation values of both $\mathrm{H}$ and $\sigma$ if $\lambda_{H}$, $\lambda_{\sigma}$ and $4 \lambda_{H} \lambda_{\sigma}-\lambda_{H \sigma}^{2}$ are all positive. The vevs break both the electroweak and lepton number symmetries, three of the degrees of freedom are eaten by the massive Standard Model gauge bosons, while the imaginary part of the $\sigma$ corresponds to the physical majoron $J=\operatorname{Im} \sigma$. The real parts of $H$ and $\sigma$ will mix with each other to give two $C P$ even mass eigenstates $h_{1}$ and $h_{2}$. The lighter of these is identified with $125 \mathrm{GeV}$ Higgs boson [1,2].

\section{VACUUM STABILITY IN TYPE-I SEESAW WITH MAJORON}

Here we take again the simplest majoron extension of the type-I seesaw mechanism based on the $(3,1)$ missing partner scheme considered above. ${ }^{8}$ We adopt the high-scale seesaw limit $v_{\sigma} \gg v_{H}$. In this limit, mass of the heavier $C P$-even scalar boson and right neutrino are approximately given as $m_{h_{2}} \equiv M \approx \sqrt{2 \lambda_{\sigma} v_{\sigma}}$ and $M_{N} \approx \frac{Y_{R}}{\sqrt{2}} v_{\sigma}$. The light and heavy Higgs sectors will be almost decoupled, though we can still allow appreciable $\lambda_{H \sigma}$ with very small mixing angle $\alpha$, see Appendix A. For simplicity, we consider nearly degenerate $M$ and $M_{N}$, such that we have only one threshold scale $\mu=M_{N}$ or $M$, below which the theory is an effective one. Above that scale we have the full theory with all the new couplings running.

When going from energies above $M$ to energies below $M$ we need to integrate out the massive scalar degree of freedom at tree level as described in Ref. [53]. This leads to a tree level threshold effect that arises from the matching conditions at the energy scale $\mu=M$. We will now briefly describe this procedure. We can write the scalar potential for the case of type-I seesaw with majoron extensions as

$$
\begin{aligned}
V_{0}= & \lambda_{H}\left(H^{\dagger} H-\frac{v_{H}^{2}}{2}\right)^{2}+\lambda_{\sigma}\left(\sigma^{\dagger} \sigma-\frac{v_{\sigma}^{2}}{2}\right)^{2} \\
& +\lambda_{H \sigma}\left(H^{\dagger} H-\frac{v_{H}^{2}}{2}\right)\left(\sigma^{\dagger} \sigma-\frac{v_{\sigma}^{2}}{2}\right) .
\end{aligned}
$$

In our case, $v_{\sigma} \gg v_{H}$, therefore $M$ is much larger than the Higgs mass $m_{h_{1}}$. As a result, below the scale $\mu=M$, we can integrate out the field $\sigma$ using the following equation of motion (apart from derivative terms)

\footnotetext{
${ }^{8}$ Note that vacuum stability in a seesaw majoron model was discussed in [52]. However, the majoron in that paper was completely detached from the neutrino sector, lacking any solid motivation. Moreover, the low scale choice for $v_{\sigma}$ was artificial, requiring tiny Dirac Yukawa couplings. In our opinion, it is best to present the discussion within a genuine low-scale neutrino mass generation mechanism, as in Ref. [10].
} 

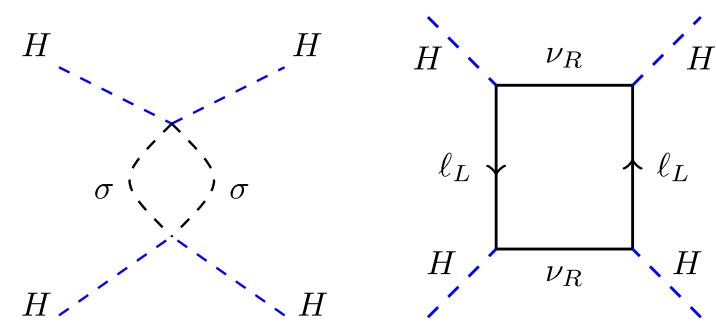

FIG. 7. One-loop correction to the Higgs quartic interaction in type-I seesaw with majoron.

$$
\begin{aligned}
& 2 \lambda_{\sigma}\left(\sigma^{\dagger} \sigma-\frac{v_{\sigma}^{2}}{2}\right)+\lambda_{H \sigma}\left(H^{\dagger} H-\frac{v_{H}^{2}}{2}\right)=0 \\
& \sigma^{\dagger} \sigma=\frac{v_{\sigma}^{2}}{2}-\frac{\lambda_{H \sigma}}{2 \lambda_{\sigma}}\left(H^{\dagger} H-\frac{v_{H}^{2}}{2}\right) .
\end{aligned}
$$

In order to obtain the effective potential below the scale $\mu=M$, we use Eq. (17) in Eq. (16), leading to the effective Higgs potential expressed as
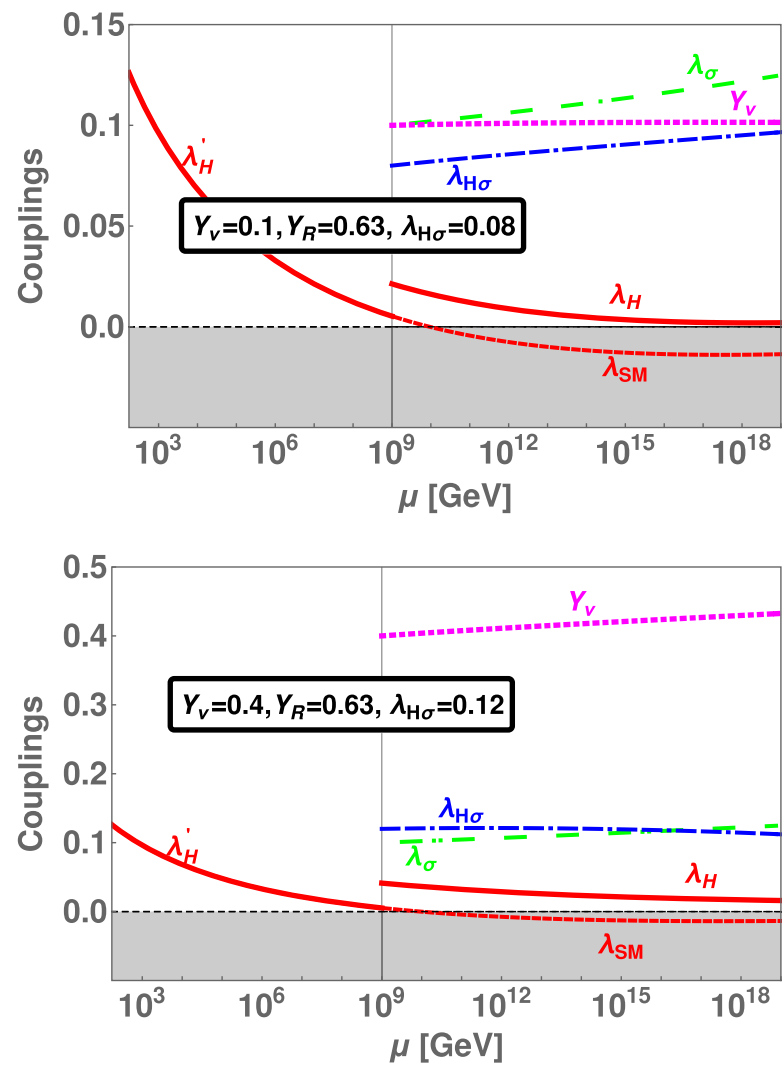

$$
V_{\text {eff }}=\lambda_{H}^{\prime}\left(H^{\dagger} H-\frac{v_{H}^{2}}{2}\right)^{2},
$$

where $\lambda_{H}^{\prime}$ is identified as

$$
\lambda_{H}^{\prime} \equiv \lambda_{\kappa}=\lambda_{H}-\frac{\lambda_{H \sigma}^{2}}{4 \lambda_{\sigma}} .
$$

Notice that, since only the dimension-five Weinberg operator is running below the scale $\mu=M$, one has that the running of $\lambda_{H}^{\prime}$ is essentially the same as that of $\lambda_{\kappa}$ in the effective type-I seesaw. Moreover, at tree-level the numerical value of $\lambda_{H}^{\prime}\left(M_{Z}\right)$ and $\lambda_{\mathrm{SM}}\left(M_{Z}\right)$ is the same, since in both cases one must reproduce the $125 \mathrm{GeV}$ Higgs mass. Equation (19) suggests that the matching condition at the scale $\mu=M$ induces a shift in the Higgs quartic coupling, $\delta \lambda=\frac{\lambda_{H \sigma}^{2}}{4 \lambda_{\sigma}}$. This corresponds to a larger Higgs quartic coupling above the scale $M$ and improves the chances of keeping $\lambda_{H}$ positive all the way.

In Appendix $\mathrm{C}$, we give the two-loop RGEs of the full theory. Note that below the scale $M$ the RGEs are the ones with $\beta_{\lambda_{H \sigma}}$ and $\beta_{\lambda_{\sigma}}$ removed and $\lambda_{H}$ replaced by $\lambda_{H}^{\prime}$. Above $M$
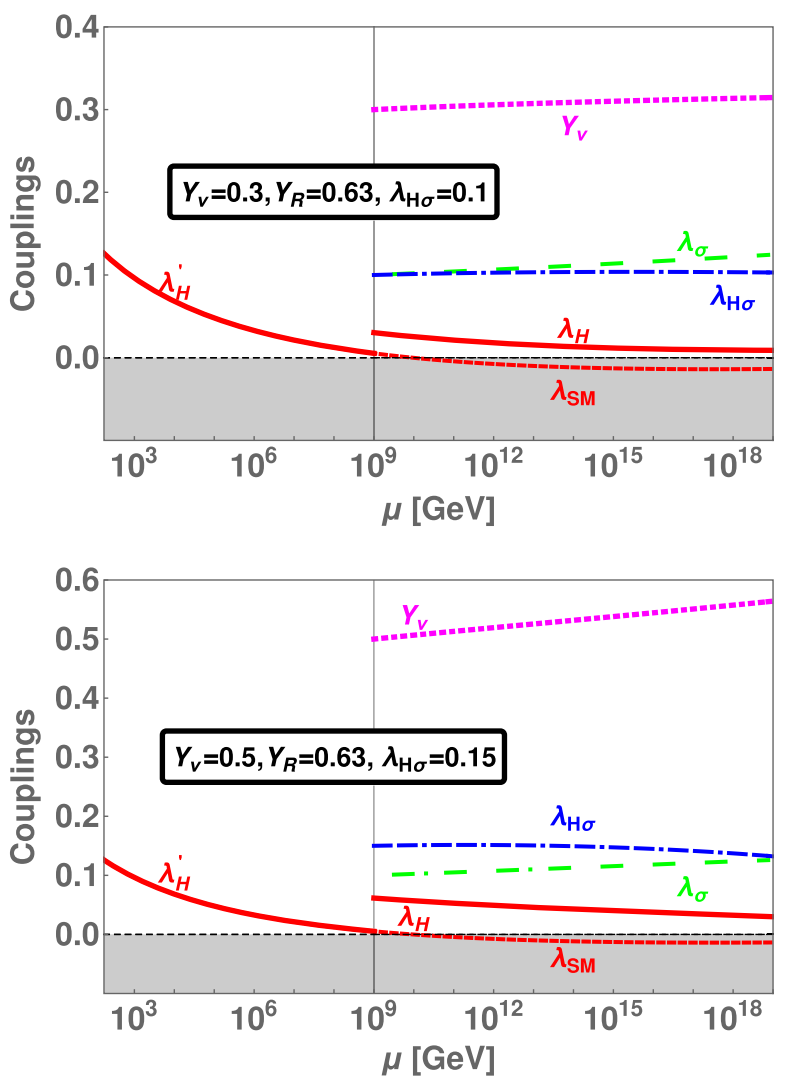

FIG. 8. Evolution of the scalar quartic couplings $\lambda_{H}, \lambda_{\sigma}$, and $\lambda_{H \sigma}$ in type-I seesaw with majoron. We include in purple dashed color the Dirac Yukawa coupling $Y_{\nu}$, see Eqs. (12) and (15). For comparison with Standard Model we have also shown the RGE for $\lambda_{\mathrm{SM}}$, red dashed curve. Here $\lambda_{H}^{\prime}$ is the effective Higgs quartic coupling below the mass threshold of the heavy particles and is essentially the same as $\lambda_{\kappa}$ in the effective type-I seesaw, see Eq. (19). Since, in this regime the RGE of $\lambda_{H}^{\prime}$ differs from that of $\lambda_{\mathrm{SM}}$ only due to the tiny contribution of the effective Weinberg operator, $\lambda_{H}^{\prime}$ and $\lambda_{\mathrm{SM}}$ almost coincide with each other. See text for more detailed discussion of various key features of the plots. 
one needs to include $\beta_{\lambda_{H \sigma}}, \beta_{\lambda_{\sigma}}$ and find $\lambda_{H}$ using the full RGEs with the boundary condition as in Eq. (19) at $\mu=M$. As far as the new Yukawa couplings are concerned, they can be obtained by substituting $Y_{\nu}=\theta\left(\mu-M_{N}\right) Y_{\nu}$ and $Y_{R}=$ $\theta(\mu-M) Y_{R}$ on the right side of the RGEs of the full theory. Figure 7 shows that positive contribution to the RGEs of the Higgs quartic coupling (left panel) is accompanied by the destabilizing effect of $\mathrm{RH}$ neutrinos through the 1-loop diagram (right panel).

Our results for the $(3,1)$ type-I seesaw mechanism with majoron are shown in Fig. 8, where we have taken $M \approx M_{N} \approx 10^{9} \mathrm{GeV}$, such that the threshold effects start contributing positively to $\lambda_{H}$ before the Standard Model instability scale $\Lambda_{\mathrm{SM}} \approx 10^{10} \mathrm{GeV}$. We have taken $\lambda_{\sigma}=0.1$ at the scale $M$. The renormalization group evolution in Fig. 8 is shown for four Yukawa coupling values $Y_{\nu}=0.1$, $0.3,0.4$, and 0.5 . It shows that, indeed, the stability properties can substantially improve due to the presence of the new scalar. In fact, for appreciable Yukawa couplings, one can have positive $\lambda_{H}$ all the way up to Planck scale. For the Yukawa couplings $Y_{\nu}=0.1,0.3,0.4$, and 0.5 , the required values of minimum $\lambda_{H \sigma}$ are $0.08,0.1,0.12$, and 0.15 , respectively.

\section{COMPARING STANDARD AND MISSING PARTNER TYPE-I SEESAW}

So far we have taken the missing partner seesaw mechanism based on the $(3,1)$ construction as our benchmark. This choice was made for the reasons given at the end of Sec. III C. Such scheme can be made phenomenologically viable in the presence of radiative corrections associated, for example, to a dark matter completion. ${ }^{9}$

Here we compare the stability properties of this simplest benchmark with those of a missing partner seesaw based on $(3,2)$ construction and with those of the standard $(3,3)$ type-I seesaw mechanism. For completeness we also compare with the Standard Model stability results.

As already mentioned, the problem of Higgs vacuum stability in type-I seesaw extensions only gets worse with the addition of extra right-handed neutrinos. This fact is clearly illustrated in Fig. 9, where we compare the evolution of the Higgs quartic self-coupling $\lambda$ within the Standard Model within the $(3, n)$ seesaw completions, with $n=1, n=2$, and $n=3$. Note that, for the general $(3, n)$ seesaw scheme $\left|Y_{\nu}\right|^{2}$ should be replaced as $\operatorname{Tr}\left(Y_{\nu}^{\dagger} Y_{\nu}\right)$ and Eq. (11) should be replaced as

$$
\lambda\left(M_{R}\right) \rightarrow \lambda\left(M_{R}\right)-\frac{5 n^{2}}{32 \pi^{2}} \operatorname{Tr}\left(Y_{\nu}^{\dagger} Y_{\nu}\right)^{2},
$$

where $n$ is the number of right handed neutrinos. For simplicity, in Fig. 9, we have fixed the benchmark

\footnotetext{
${ }^{9}$ An explicit scotogenic model of this type has been proposed in [13].
}

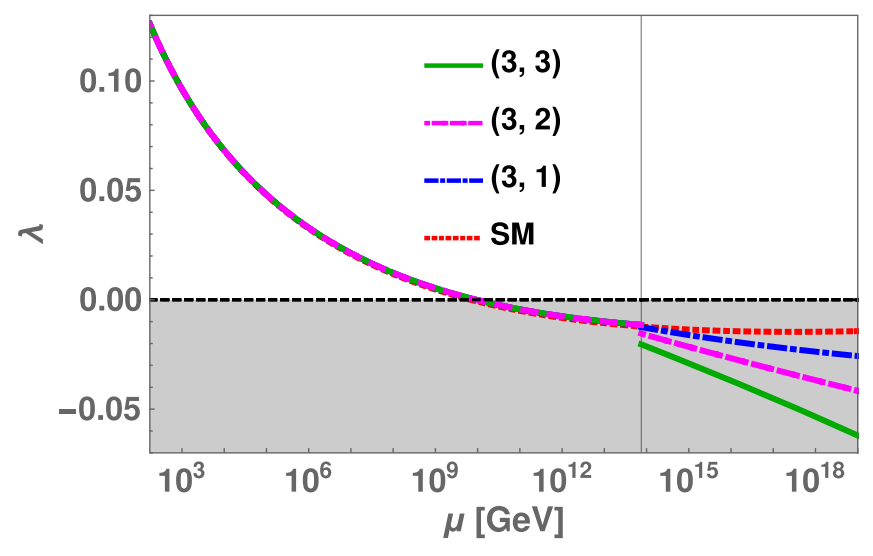

FIG. 9. Zoomed view of the evolution of the quartic Higgs selfcoupling $\lambda$ in the Standard Model (red-dashed) and in the $(3,1)$, $(3,2)$, and $(3,3)$ seesaw extensions (blue dot-dashed, magentadashed, and green solid, respectively). In the $(3,1)$ case we have taken $Y_{\nu}=0.5$, while for $(3, n=2,3)$ we took $Y_{\nu}^{a j}=0.5$; $a=$ $j=1, \ldots, n$ and $Y_{\nu}^{a j}=0$ for $a \neq j$.

value of $Y_{\nu}^{a j}=0.5 ; a=j=1,2,3$ and taken the offdiagonal terms to be zero for the $(3,3)$ case. Note that such a choice is unrealistic vis-a-vis neutrino oscillation data. However, taking the Yukawa texture consistent with neutrino oscillation data will not change our conclusions. Therefore, for the sake of simplicity, we have taken this simple choice.

In contrast, going to the $(3,3)$ majoron type-I seesaw with three right handed neutrinos, we find that the Higgs vacuum can be still kept stable up to Planck scale for appreciable Yukawa couplings. Of course, the presence of additional fermions means that the maximum values of $Y_{\nu}^{a i}$, for which Higgs vacuum stability can be achieved up to Planck scale, is somewhat reduced. In Fig. 10 we compare the Higgs

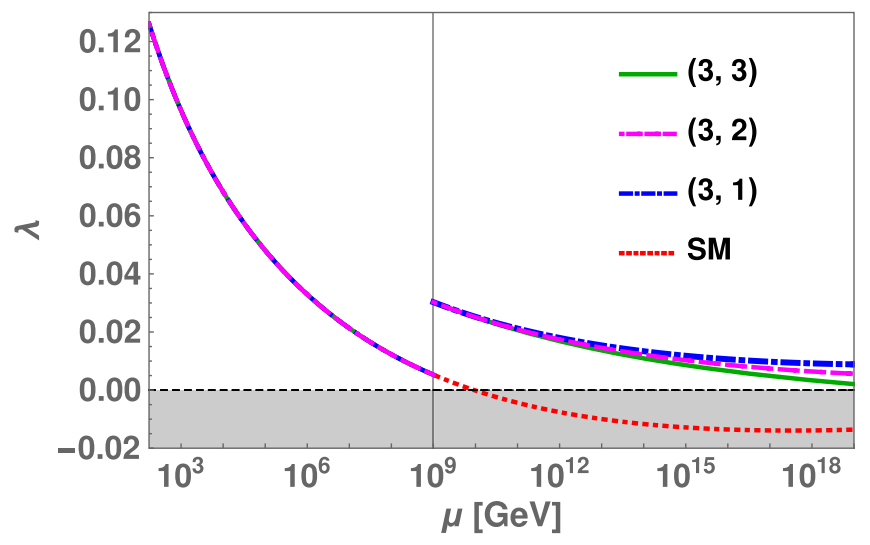

FIG. 10. Zoomed view of the evolution of the quartic Higgs self-coupling $\lambda$ in the Standard Model (red-dashed) and $(3,1)$, $(3,2)$, and $(3,3)$ majoron seesaw (blue dot-dashed, magentadashed, and green solid, respectively). In the $(3,1)$ case we have taken $Y_{\nu}=0.3$, while for $(3, n=2,3)$ we took $Y_{\nu}^{a j}=0.3$; $a=$ $j=1, \ldots, n$ and $Y_{\nu}^{a j}=0$ for $a \neq j$. 
vacuum stability of the $(3,3)$ majoron seesaw case with its $(3,1)$ analogue as well as with Standard Model.

For Fig. 10 we have taken $Y_{\nu}=0.3$ for the $(3,1)$ case, while for the $(3,3)$ case we have taken $Y_{\nu}^{a i}=0.3$; $a=i=1,2,3$, with all the off-diagonal entries taken to zero. The remaining parameters are kept the same as described previously for the $(3,1)$ majoron seesaw case. In short, phenomenologically realistic type-I seesaw majoron models can have a stable vacuum all the way up to Planck scale.

\section{SUMMARY AND OUTLOOK}

We have examined the consistency of electroweak symmetry breaking within the context of the simplest high-scale type-I seesaw mechanism. We have derived the full two-loop RGEs for the relevant parameters, such as the quartic Higgs self-coupling $\lambda$ of the Standard Model within the schemes of interest. These are compared, for calibration, with the Standard Model results. The addition of fermionic fields like "right-handed" neutrinos, has a destabilizing effect on the Higgs boson vacuum. For the simplest type-I seesaw with bare mass term for the righthanded neutrinos, we found that for sizeable Yukawa couplings the Higgs quartic self-coupling $\lambda$ becomes negative much before reaching the seesaw scale. For such "large" Yukawas the type-I seesaw may be inconsistent even as an effective theory. We have taken as our simplest benchmark neutrino model the "incomplete" $(3,1)$ seesaw scheme with a single right-handed neutrino, as it has the "best" stability properties within the class of high-scale type-I seesaw schemes. We compared this case, in which only one oscillation scale is generated at tree level, with the "higher" $(3,2)$ type-I seesaw, in which the other mass scale also arises from the tree level seesaw mechanism. In both "missing partner" type-I seesaw schemes, $(3,1)$ and $(3,2)$, the neutrinoless double beta decay prediction given in Fig. 2 holds. We also studied the stability of the electroweak vacuum for the canonical sequential $(3,3)$ type-I seesaw, in which all three neutrinos get tree-level mass. We showed how the stability properties improve in the case of spontaneous lepton number violation due to the presence of a Nambu-Goldstone boson, the majoron.

To sum up, our results show how, in contrast to the type-I seesaw with explicit breaking of a lepton number, the majoron version can have stable electroweak vacuum all the way up to Planck scale for reasonable Yukawa coupling choices. Thus, the majoron completion of type-I seesaw schemes can be considered as fully consistent theories.

Before concluding we should note the cosmological advantages of the majoron completion. The first is that it can also provide a dark matter candidate, namely the majoron [17], providing an alternative to the $\Lambda \mathrm{CDM}$ paradigm. The majoron is assumed to get mass from gravitational effects that explicitly violate the global lepton number [54]. Assuming that its mass lies in the $\mathrm{keV}$ range one can show that it can provide a viable warm dark matter candidate. It decays to neutrinos, with a tiny strength proportional to their mass [16]. Hence, it is naturally long-lived on a cosmological scale, as required, with lifetime $\tau_{J}$ larger than the age of the Universe $t_{0}=13.8 \mathrm{Gyr} \simeq 4 \times 10^{17} \mathrm{~s}$. Such majoron dark matter scenario has been shown to be consistent with cosmic microwave background data for adequate choices of the relevant parameters $[18,20,55]$, the majoron decay lifetime constraints ranging from $\tau_{J}>50-160 \mathrm{Gyr}$. Using N-body simulations one can also show that majoron dark matter provides a viable alternative to the $\Lambda \mathrm{CDM}$ scenario, with predictions that can differ substantially on small scales [21]. Finally we also mention that, in addition to dark matter, the majoron picture may also provide new insights to other cosmological challenges of the Standard Model, such as inflation [56] and leptogenesis [57].

\section{ACKNOWLEDGMENTS}

This work is supported by the Spanish Grants No. FPA2017-85216-P (AEI/FEDER, UE), No. PROMETEO/ 2018/165 (Generalitat Valenciana), and the Spanish Red Consolider MultiDark FPA2017-90566-REDC. We thank Martin Hirsch and Werner Porod for useful discussions.

\section{APPENDIX A: HIGGS SECTOR IN MAJORON MODEL}

The scalar potential for the majoron type-I seesaw is given by,

$$
\begin{aligned}
V= & -\mu_{H}^{2} H^{\dagger} H-\mu_{\sigma}^{2} \sigma^{\dagger} \sigma+\lambda_{H}\left(H^{\dagger} H\right)^{2}+\lambda_{\sigma}\left(\sigma^{\dagger} \sigma\right)^{2} \\
& +\lambda_{H \sigma}\left(H^{\dagger} H\right)\left(\sigma^{\dagger} \sigma\right) .
\end{aligned}
$$

The Standard Model gauge singlet scalar $\sigma$ carries two units of lepton number and its vev $\langle\sigma\rangle=\frac{v_{\sigma}}{\sqrt{2}}$ breaks the lepton number symmetry $U(1)_{L}$ to a $\mathbb{Z}_{2}$ subgroup. After symmetry breaking one has, in the unitary gauge

$$
H \rightarrow \frac{1}{\sqrt{2}}\left(\begin{array}{c}
0 \\
v_{H}+h^{\prime}
\end{array}\right), \quad \sigma \rightarrow \frac{v_{\sigma}+\sigma^{\prime}}{\sqrt{2}} .
$$

The scalars $h^{\prime}$ and $\sigma^{\prime}$ will mix with each other, their mass eigenvalues are given by,

$m_{h_{1}}^{2}=\lambda_{H} v_{H}^{2}+\lambda_{\sigma} v_{\sigma}^{2}-\sqrt{\left(\lambda_{H} v_{H}^{2}-\lambda_{\sigma} v_{\sigma}^{2}\right)^{2}+\left(\lambda_{H \sigma} v_{H} v_{\sigma}\right)^{2}}$,

$m_{h_{2}}^{2}=\lambda_{H} v_{H}^{2}+\lambda_{\sigma} v_{\sigma}^{2}+\sqrt{\left(\lambda_{H} v_{H}^{2}-\lambda_{\sigma} v_{\sigma}^{2}\right)^{2}+\left(\lambda_{H \sigma} v_{H} v_{\sigma}\right)^{2}}$.

The mass eigenstates $h_{1}, h_{2}$ are related to the fields $h^{\prime}, \sigma^{\prime}$ by the mixing matrix parameterized by the angle $\alpha$ and given by 


$$
\left(\begin{array}{l}
h_{1} \\
h_{2}
\end{array}\right)=\left(\begin{array}{cc}
\cos \alpha & -\sin \alpha \\
\sin \alpha & \cos \alpha
\end{array}\right)\left(\begin{array}{l}
h^{\prime} \\
\sigma^{\prime}
\end{array}\right),
$$

where the mixing angle $\alpha$ is given by,

$$
\begin{aligned}
\sin 2 \alpha & =\frac{\lambda_{H \sigma} v_{H} v_{\sigma}}{\sqrt{\left(\lambda_{H} v_{H}^{2}-\lambda_{\sigma} v_{\sigma}^{2}\right)^{2}+\left(\lambda_{H \sigma} v_{H} v_{\sigma}\right)^{2}}}, \\
\cos 2 \alpha & =\frac{\lambda_{H} v_{H}^{2}-\lambda_{\sigma} v_{\sigma}^{2}}{\sqrt{\left(\lambda_{H} v_{H}^{2}-\lambda_{\sigma} v_{\sigma}^{2}\right)^{2}+\left(\lambda_{H \sigma} v_{H} v_{\sigma}\right)^{2}}} .
\end{aligned}
$$

One can see from (A6) that in the limit $v_{\sigma} \gg v_{H}$ the mixing angle $\alpha \rightarrow 0$, irrespective of the value of the quartic couplings.

\section{APPENDIX B: RGES: TYPE I SEESAW}

We have used the package SARAH [58] to do the RGEs analysis in our work. The $\beta$ function of a given parameter $\mathrm{c}$ is given by

$$
\frac{d c}{d t} \equiv \beta_{c}=\frac{1}{16 \pi^{2}} \beta_{c}^{(1)}+\frac{1}{\left(16 \pi^{2}\right)^{2}} \beta_{c}^{(2)},
$$

where $\beta_{c}^{(1)}$ are the one-loop RGEs corrections and $\beta_{c}^{(2)}$ are the two-loop RGEs corrections.

\section{Higgs quartic scalar self coupling}

For the most general $(3, n)$ seesaw the one-loop and twoloop RGEs corrections to the Higgs quartic self-coupling are given by:

$$
\begin{aligned}
\beta_{\lambda}^{(1)}= & +\frac{27}{200} g_{1}^{4}+\frac{9}{20} g_{1}^{2} g_{2}^{2}+\frac{9}{8} g_{2}^{4}-\frac{9}{5} g_{1}^{2} \lambda-9 g_{2}^{2} \lambda+24 \lambda^{2} \\
& +12 \lambda y_{t}^{2}+4 \lambda \operatorname{Tr}\left(Y_{\nu} Y_{\nu}^{\dagger}\right)-6 y t^{4}-2 \operatorname{Tr}\left(Y_{\nu} Y_{\nu}^{\dagger} Y_{\nu} Y_{\nu}^{\dagger}\right),
\end{aligned}
$$

$$
\begin{aligned}
\beta_{\lambda}^{(2)}= & -\frac{3411}{2000} g_{1}^{6}-\frac{1677}{400} g_{1}^{4} g_{2}^{2}-\frac{289}{80} g_{1}^{2} g_{2}^{4}+\frac{305}{16} g_{2}^{6}+\frac{1887}{200} g_{1}^{4} \lambda+\frac{117}{20} g_{1}^{2} g_{2}^{2} \lambda-\frac{73}{8} g_{2}^{4} \lambda+\frac{108}{5} g_{1}^{2} \lambda^{2} \\
& +108 g_{2}^{2} \lambda^{2}-312 \lambda^{3}-\frac{171}{100} g_{1}^{4} y_{t}^{2}+\frac{63}{10} g_{1}^{2} g_{2}^{2} y_{t}^{2}-\frac{9}{4} g_{2}^{4} y_{t}^{2}+\frac{17}{2} g_{1}^{2} \lambda y_{t}^{2}+\frac{45}{2} g_{2}^{2} \lambda y_{t}^{2}+80 g_{3}^{2} \lambda y_{t}^{2}-144 \lambda^{2} y_{t}^{2} \\
& -\frac{9}{100} g_{1}^{4} \operatorname{Tr}\left(Y_{\nu} Y_{\nu}^{\dagger}\right)-\frac{3}{10} g_{1}^{2} g_{2}^{2} \operatorname{Tr}\left(Y_{\nu} Y_{\nu}^{\dagger}\right)-\frac{3}{4} g_{2}^{4} \operatorname{Tr}\left(Y_{\nu} Y_{\nu}^{\dagger}\right)+\frac{3}{2} g_{1}^{2} \lambda \operatorname{Tr}\left(Y_{\nu} Y_{\nu}^{\dagger}\right)+\frac{15}{2} g_{2}^{2} \lambda \operatorname{Tr}\left(Y_{\nu} Y_{\nu}^{\dagger}\right)-48 \lambda^{2} \operatorname{Tr}\left(Y_{\nu} Y_{\nu}^{\dagger}\right) \\
& -\frac{8}{5} g_{1}^{2} y_{t}^{4}-32 g_{3}^{2} y_{t}^{4}-3 \lambda y_{t}^{4}-\lambda \operatorname{Tr}\left(Y_{\nu} Y_{\nu}^{\dagger} Y_{\nu} Y_{\nu}^{\dagger}\right)+30 y_{t}^{6}+10 \operatorname{Tr}\left(Y_{\nu} Y_{\nu}^{\dagger} Y_{\nu} Y_{\nu}^{\dagger} Y_{\nu} Y_{\nu}^{\dagger}\right) .
\end{aligned}
$$

\section{Yukawa Couplings}

The one-loop and two-loop RGEs corrections to $Y_{\nu}$ in the $(3, \mathrm{n})$ seesaw are given by

$$
\begin{gathered}
\beta_{Y_{\nu}}^{(1)}=\frac{3}{2} Y_{\nu} Y_{\nu}^{\dagger} Y_{\nu}+Y_{\nu}\left(3 y_{t}^{2}-\frac{9}{20} g_{1}^{2}-\frac{9}{4} g_{2}^{2}+\operatorname{Tr}\left(Y_{\nu} Y_{\nu}^{\dagger}\right)\right), \\
\beta_{Y_{\nu}}^{(2)}=\frac{1}{80}\left(279 g_{1}^{2} Y_{\nu} Y_{\nu}^{\dagger} Y_{\nu}+675 g_{2}^{2} Y_{\nu} Y_{\nu}^{\dagger} Y_{\nu}-960 \lambda Y_{\nu} Y_{\nu}^{\dagger} Y_{\nu}+120 Y_{\nu} Y_{\nu}^{\dagger} Y_{\nu} Y_{\nu}^{\dagger} Y_{\nu}-540 Y_{\nu} Y_{\nu}^{\dagger} Y_{\nu} y_{t}^{2}\right. \\
-180 Y_{\nu} Y_{\nu}^{\dagger} Y_{\nu} \operatorname{Tr}\left(Y_{\nu} Y_{\nu}^{\dagger}\right)+2 Y_{\nu}\left(21 g_{1}^{4}-54 g_{1}^{2} g_{2}^{2}-230 g_{2}^{4}+240 \lambda^{2}+85 g_{1}^{2} y_{t}^{2}+225 g_{2}^{2} y_{t}^{2}\right. \\
\left.\left.+800 g_{3}^{2} y_{t}^{2}+15 g_{1}^{2} \operatorname{Tr}\left(Y_{\nu} Y_{\nu}^{\dagger}\right)+75 g_{2}^{2} \operatorname{Tr}\left(Y_{\nu} Y_{\nu}^{\dagger}\right)-270 y_{t}^{4}-90 \operatorname{Tr}\left(Y_{\nu} Y_{\nu}^{\dagger} Y_{\nu} Y_{\nu}^{\dagger}\right)\right)\right) .
\end{gathered}
$$

The RGEs corrections to the top-Yukawa coupling $y_{t}$ are given by

$$
\begin{gathered}
\beta_{y_{t}}^{(1)}=\frac{3}{2} y_{t}^{3}+y_{t}\left(3 y_{t}^{2}-8 g_{3}^{2}-\frac{17}{20} g_{1}^{2}-\frac{9}{4} g_{2}^{2}+\operatorname{Tr}\left(Y_{\nu} Y_{\nu}^{\dagger}\right)\right) \\
\beta_{y_{t}}^{(2)}=+\frac{1}{80}\left(120 y_{t}^{5}+y_{t}^{3}\left(1280 g_{3}^{2}-180 \operatorname{Tr}\left(Y_{\nu} Y_{\nu}^{\dagger}\right)+223 g_{1}^{2}-540 y_{t}^{2}+675 g_{2}^{2}-960 \lambda\right)\right) \\
+y_{t}\left(\frac{1187}{600} g_{1}^{4}-\frac{9}{20} g_{1}^{2} g_{2}^{2}-\frac{23}{4} g_{2}^{4}+\frac{19}{15} g_{1}^{2} g_{3}^{2}+9 g_{2}^{2} g_{3}^{2}-108 g_{3}^{4}+6 \lambda^{2}+\frac{17}{8} g_{1}^{2} y_{t}^{2}+\frac{45}{8} g_{2}^{2} y_{t}^{2}\right. \\
\left.+20 g_{3}^{2} y_{t}^{2}+\frac{3}{8} g_{1}^{2} \operatorname{Tr}\left(Y_{\nu} Y_{\nu}^{\dagger}\right)+\frac{15}{8} g_{2}^{2} \operatorname{Tr}\left(Y_{\nu} Y_{\nu}^{\dagger}\right)-\frac{27}{4} y_{t}^{4}-\frac{9}{4} \operatorname{Tr}\left(Y_{\nu} Y_{\nu}^{\dagger} Y_{\nu} Y_{\nu}^{\dagger}\right)\right) .
\end{gathered}
$$




\section{APPENDIX C: RGES: TYPE I SEESAW WITH MAJORON}

\section{Quartic scalar couplings}

The scalar sector of the majoron model is given in Eq. (15). It contains three scalar quartic couplings $\lambda_{H}, \lambda_{H \sigma}, \lambda_{\sigma}$ whose one-loop and two-loop RGEs are given by

$$
\begin{aligned}
& \beta_{\lambda_{H}}^{(1)}=+\frac{27}{200} g_{1}^{4}+\frac{9}{20} g_{1}^{2} g_{2}^{2}+\frac{9}{8} g_{2}^{4}+\lambda_{H \sigma}^{2}-\frac{9}{5} g_{1}^{2} \lambda_{H}-9 g_{2}^{2} \lambda_{H}+24 \lambda_{H}^{2}+12 \lambda_{H} y_{t}^{2}+4 \lambda_{H} \operatorname{Tr}\left(Y_{\nu} Y_{\nu}^{\dagger}\right)-6 y_{t}^{4}-2 \operatorname{Tr}\left(Y_{\nu} Y_{\nu}^{\dagger} Y_{\nu} Y_{\nu}^{\dagger}\right), \\
& \beta_{\lambda_{H}}^{(2)}=-\frac{3411}{2000} g_{1}^{6}-\frac{1677}{400} g_{1}^{4} g_{2}^{2}-\frac{289}{80} g_{1}^{2} g_{2}^{4}+\frac{305}{16} g_{2}^{6}-4 \lambda_{H \sigma}^{3}+\frac{1887}{200} g_{1}^{4} \lambda_{H}+\frac{117}{20} g_{1}^{2} g_{2}^{2} \lambda_{H}-\frac{73}{8} g_{2}^{4} \lambda_{H} \\
& -10 \lambda_{H \sigma}^{2} \lambda_{H}+\frac{108}{5} g_{1}^{2} \lambda_{H}^{2}+108 g_{2}^{2} \lambda_{H}^{2}-312 \lambda_{H}^{3}-\lambda_{H \sigma}^{2} \operatorname{Tr}\left(Y_{R} Y_{R}^{*}\right)-\frac{171}{100} g_{1}^{4} y_{t}^{2}+\frac{63}{10} g_{1}^{2} g_{2}^{2} y_{t}^{2} \\
& -\frac{9}{4} g_{2}^{4} y_{t}^{2}+\frac{17}{2} g_{1}^{2} \lambda_{H} y_{t}^{2}+\frac{45}{2} g_{2}^{2} \lambda_{H} y_{t}^{2}+80 g_{3}^{2} \lambda_{H} y_{t}^{2}-144 \lambda_{H}^{2} y_{t}^{2}-\frac{9}{100} g_{1}^{4} \operatorname{Tr}\left(Y_{\nu} Y_{\nu}^{\dagger}\right)-\frac{3}{10} g_{1}^{2} g_{2}^{2} \operatorname{Tr}\left(Y_{\nu} Y_{\nu}^{\dagger}\right) \\
& -\frac{3}{4} g_{2}^{4} \operatorname{Tr}\left(Y_{\nu} Y_{\nu}^{\dagger}\right)+\frac{3}{2} g_{1}^{2} \lambda_{H} \operatorname{Tr}\left(Y_{\nu} Y_{\nu}^{\dagger}\right)+\frac{15}{2} g_{2}^{2} \lambda_{H} \operatorname{Tr}\left(Y_{\nu} Y_{\nu}^{\dagger}\right)-48 \lambda_{H}^{2} \operatorname{Tr}\left(Y_{\nu} Y_{\nu}^{\dagger}\right)-3 \lambda_{H} \operatorname{Tr}\left(Y_{R} Y_{\nu}^{\dagger} Y_{\nu} Y_{R}^{*}\right) \\
& -\frac{8}{5} g_{1}^{2} y_{t}^{4}-32 g_{3}^{2} y_{t}^{4}-3 \lambda_{H} y_{t}^{4}-\lambda_{H} \operatorname{Tr}\left(Y_{\nu} Y_{\nu}^{\dagger} Y_{\nu} Y_{\nu}^{\dagger}\right)+2 \operatorname{Tr}\left(Y_{R} Y_{\nu}^{\dagger} Y_{\nu} Y_{\nu}^{\dagger} Y_{\nu} Y_{R}^{*}\right)+2 \operatorname{Tr}\left(Y_{R} Y_{\nu}^{\dagger} Y_{\nu} Y_{R}^{*} Y_{\nu}^{T} Y_{\nu}^{*}\right) \\
& +30 y_{t}^{6}+10 \operatorname{Tr}\left(Y_{\nu} Y_{\nu}^{\dagger} Y_{\nu} Y_{\nu}^{\dagger} Y_{\nu} Y_{\nu}^{\dagger}\right) \\
& \beta_{\lambda_{H \sigma}}^{(1)}=-\frac{9}{10} g_{1}^{2} \lambda_{H \sigma}-\frac{9}{2} g_{2}^{2} \lambda_{H \sigma}+4 \lambda_{H \sigma}^{2}+8 \lambda_{H \sigma} \lambda_{\sigma}+12 \lambda_{H \sigma} \lambda_{H}+\lambda_{H \sigma} \operatorname{Tr}\left(Y_{R} Y_{R}^{*}\right)+2 \lambda_{H \sigma} \operatorname{Tr}\left(Y_{\nu} Y_{\nu}^{\dagger}\right) \\
& +6 \lambda_{H \sigma} y_{t}^{2}-4 \operatorname{Tr}\left(Y_{R} Y_{\nu}^{\dagger} Y_{\nu} Y_{R}^{*}\right) \text {, } \\
& \beta_{\lambda_{H \sigma}}^{(2)}=+\frac{1671}{400} g_{1}^{4} \lambda_{H \sigma}+\frac{9}{8} g_{1}^{2} g_{2}^{2} \lambda_{H \sigma}-\frac{145}{16} g_{2}^{4} \lambda_{H \sigma}+\frac{3}{5} g_{1}^{2} \lambda_{H \sigma}^{2}+3 g_{2}^{2} \lambda_{H \sigma}^{2}-11 \lambda_{H \sigma}^{3}-48 \lambda_{H \sigma}^{2} \lambda_{\sigma}-40 \lambda_{H \sigma} \lambda_{\sigma}^{2} \\
& +\frac{72}{5} g_{1}^{2} \lambda_{H \sigma} \lambda_{H}+72 g_{2}^{2} \lambda_{H \sigma} \lambda-72 \lambda_{H \sigma}^{2} \lambda_{H}-60 \lambda_{H \sigma} \lambda_{H}^{2}-2 \lambda_{H \sigma}^{2} \operatorname{Tr}\left(Y_{R} Y_{R}^{*}\right)-8 \lambda_{H \sigma} \lambda_{\sigma} \operatorname{Tr}\left(Y_{R} Y_{R}^{*}\right) \\
& +\frac{17}{4} g_{1}^{2} \lambda_{H \sigma} y_{t}^{2}+\frac{45}{4} g_{2}^{2} \lambda_{H \sigma} y_{t}^{2}+40 g_{3}^{2} \lambda_{H \sigma} y_{t}^{2}-12 \lambda_{H \sigma}^{2} y_{t}^{2}-72 \lambda_{H \sigma} \lambda_{H} y_{t}^{2}+\frac{3}{4} g_{1}^{2} \lambda_{H \sigma} \operatorname{Tr}\left(Y_{\nu} Y_{\nu}^{\dagger}\right) \\
& +\frac{15}{4} g_{2}^{2} \lambda_{H \sigma} \operatorname{Tr}\left(Y_{\nu} Y_{\nu}^{\dagger}\right)-4 \lambda_{H \sigma}^{2} \operatorname{Tr}\left(Y_{\nu} Y_{\nu}^{\dagger}\right)-24 \lambda_{H \sigma} \lambda_{H} \operatorname{Tr}\left(Y_{\nu} Y_{\nu}^{\dagger}\right)+\frac{7}{2} \lambda_{H \sigma} \operatorname{Tr}\left(Y_{R} Y_{\nu}^{\dagger} Y_{\nu} Y_{R}^{*}\right) \\
& -\frac{3}{2} \lambda_{H \sigma} \operatorname{Tr}\left(Y_{R} Y_{R}^{*} Y_{R} Y_{R}^{*}\right)-\frac{27}{2} \lambda_{H \sigma} y_{t}^{4}-\frac{9}{2} \lambda_{H \sigma} \operatorname{Tr}\left(Y_{\nu} Y_{\nu}^{\dagger} Y_{\nu} Y_{\nu}^{\dagger}\right)+14 \operatorname{Tr}\left(Y_{R} Y_{\nu}^{\dagger} Y_{\nu} Y_{\nu}^{\dagger} Y_{\nu} Y_{R}^{*}\right) \\
& +\frac{41}{8} \operatorname{Tr}\left(Y_{R} Y_{\nu}^{\dagger} Y_{\nu} Y_{R}^{*} Y_{R} Y_{R}^{*}\right)+8 \operatorname{Tr}\left(Y_{R} Y_{\nu}^{\dagger} Y_{\nu} Y_{R}^{*} Y_{\nu}^{T} Y_{\nu}^{*}\right)+\frac{39}{8} \operatorname{Tr}\left(Y_{R} Y_{R}^{*} Y_{R} Y_{\nu}^{\dagger} Y_{\nu} Y_{R}^{*}\right), \\
& \beta_{\lambda_{\sigma}}^{(1)}=20 \lambda_{\sigma}^{2}+2 \lambda_{H \sigma}^{2}+2 \lambda_{\sigma} \operatorname{Tr}\left(Y_{R} Y_{R}^{*}\right)-\operatorname{Tr}\left(Y_{R} Y_{R}^{*} Y_{R} Y_{R}^{*}\right) \\
& \beta_{\lambda_{\sigma}}^{(2)}=+\frac{12}{5} g_{1}^{2} \lambda_{H \sigma}^{2}+12 g_{2}^{2} \lambda_{H \sigma}^{2}-8 \lambda_{H \sigma}^{3}-20 \lambda_{H \sigma}^{2} \lambda_{\sigma}-240 \lambda_{\sigma}^{3}-20 \lambda_{\sigma}^{2} \operatorname{Tr}\left(Y_{R} Y_{R}^{*}\right)-12 \lambda_{H \sigma}^{2} y_{t}^{2} \\
& -4 \lambda_{H \sigma}^{2} \operatorname{Tr}\left(Y_{\nu} Y_{\nu}^{\dagger}\right)-6 \lambda_{\sigma} \operatorname{Tr}\left(Y_{R} Y_{\nu}^{\dagger} Y_{\nu} Y_{R}^{*}\right)+\lambda_{\sigma} \operatorname{Tr}\left(Y_{R} Y_{R}^{*} Y_{R} Y_{R}^{*}\right)+\frac{13}{4} \operatorname{Tr}\left(Y_{R} Y_{\nu}^{\dagger} Y_{\nu} Y_{R}^{*} Y_{R} Y_{R}^{*}\right) \\
& +\frac{3}{4} \operatorname{Tr}\left(Y_{R} Y_{R}^{*} Y_{R} Y_{\nu}^{\dagger} Y_{\nu} Y_{R}^{*}\right)+4 \operatorname{Tr}\left(Y_{R} Y_{R}^{*} Y_{R} Y_{R}^{*} Y_{R} Y_{R}^{*}\right) .
\end{aligned}
$$




\section{Yukawa Couplings}

The one-loop and two-loop RGEs of the Yukawa couplings $Y_{\nu}, y_{t}$ and $Y_{R}$ are given by

$$
\begin{aligned}
& \beta_{Y_{\nu}}^{(1)}=+\frac{1}{2}\left(3 Y_{\nu} Y_{\nu}^{\dagger} Y_{\nu}+Y_{\nu} Y_{R}^{*} Y_{R}\right)+Y_{\nu}\left(3 y_{t}^{2}-\frac{9}{20} g_{1}^{2}-\frac{9}{4} g_{2}^{2}+\operatorname{Tr}\left(Y_{\nu} Y_{\nu}^{\dagger}\right)\right), \\
& \beta_{Y_{\nu}}^{(2)}=\frac{1}{80}\left(279 g_{1}^{2} Y_{\nu} Y_{\nu}^{\dagger} Y_{\nu}+675 g_{2}^{2} Y_{\nu} Y_{\nu}^{\dagger} Y_{\nu}-960 \lambda_{H} Y_{\nu} Y_{\nu}^{\dagger} Y_{\nu}-160 \lambda_{H \sigma} Y_{\nu} Y_{R}^{*} Y_{R}+120 Y_{\nu} Y_{\nu}^{\dagger} Y_{\nu} Y_{\nu}^{\dagger} Y_{\nu}-10 Y_{\nu} Y_{R}^{*} Y_{R} Y_{\nu}^{\dagger} Y_{\nu}\right. \\
& -10 Y_{\nu} Y_{R}^{*} Y_{R} Y_{R}^{*} Y_{R}+140 Y_{\nu} Y_{R}^{*} Y_{\nu}^{T} Y_{\nu}^{*} Y_{R}-30 Y_{\nu} Y_{R}^{*} Y_{R} \operatorname{Tr}\left(Y_{R} Y_{R}^{*}\right)-540 Y_{\nu} Y_{\nu}^{\dagger} Y_{\nu} y_{t}^{2}-180 Y_{\nu} Y_{\nu}^{\dagger} Y_{\nu} \operatorname{Tr}\left(Y_{\nu} Y_{\nu}^{\dagger}\right) \\
& +2 Y_{\nu}\left(21 g_{1}^{4}-54 g_{1}^{2} g_{2}^{2}-230 g_{2}^{4}+20 \lambda_{H \sigma}^{2}+240 \lambda_{H}^{2}+85 g_{1}^{2} y_{t}^{2}+225 g_{2}^{2} y_{t}^{2}+800 g_{3}^{2} y_{t}^{2}+15 g_{1}^{2} \operatorname{Tr}\left(Y_{\nu} Y_{\nu}^{\dagger}\right)\right. \\
& \left.\left.+75 g_{2}^{2} \operatorname{Tr}\left(Y_{\nu} Y_{\nu}^{\dagger}\right)-30 \operatorname{Tr}\left(Y_{R} Y_{\nu}^{\dagger} Y_{\nu} Y_{R}^{*}\right)-270 y_{t}^{4}-90 \operatorname{Tr}\left(Y_{\nu} Y_{\nu}^{\dagger} Y_{\nu} Y_{\nu}^{\dagger}\right)\right)\right) \text {, } \\
& \beta_{y_{t}}^{(1)}=\frac{3}{2} y_{t}^{3}+y_{t}\left(3 y_{t}^{2}-8 g_{3}^{2}-\frac{17}{20} g_{1}^{2}-\frac{9}{4} g_{2}^{2}+\operatorname{Tr}\left(Y_{\nu} Y_{\nu}^{\dagger}\right)\right) \\
& \beta_{y_{t}}^{(2)}=+\frac{1}{80}\left(120 y_{t}^{5}+y_{t}^{3}\left(1280 g_{3}^{2}-180 \operatorname{Tr}\left(Y_{\nu} Y_{\nu}^{\dagger}\right)+223 g_{1}^{2}-540 y_{t}^{2}+675 g_{2}^{2}-960 \lambda_{H}\right)\right) \\
& +y_{t}\left(\frac{1187}{600} g_{1}^{4}-\frac{9}{20} g_{1}^{2} g_{2}^{2}-\frac{23}{4} g_{2}^{4}+\frac{19}{15} g_{1}^{2} g_{3}^{2}+9 g_{2}^{2} g_{3}^{2}-108 g_{3}^{4}+\frac{1}{2} \lambda_{H \sigma}^{2}+6 \lambda_{H}^{2}+\frac{17}{8} g_{1}^{2} y_{t}^{2}\right. \\
& \left.+\frac{45}{8} g_{2}^{2} y_{t}^{2}+20 g_{3}^{2} y_{t}^{2}+\frac{3}{8} g_{1}^{2} \operatorname{Tr}\left(Y_{\nu} Y_{\nu}^{\dagger}\right)+\frac{15}{8} g_{2}^{2} \operatorname{Tr}\left(Y_{\nu} Y_{\nu}^{\dagger}\right)-\frac{3}{4} \operatorname{Tr}\left(Y_{R} Y_{\nu}^{\dagger} Y_{\nu} Y_{R}^{*}\right)-\frac{27}{4} y_{t}^{4}-\frac{9}{4} \operatorname{Tr}\left(Y_{\nu} Y_{\nu}^{\dagger} Y_{\nu} Y_{\nu}^{\dagger}\right)\right), \\
& \beta_{Y_{R}}^{(1)}=\frac{1}{2} Y_{R} \operatorname{Tr}\left(Y_{R} Y_{R}^{*}\right)+Y_{R} Y_{\nu}^{\dagger} Y_{\nu}+Y_{R} Y_{R}^{*} Y_{R}+Y_{\nu}^{T} Y_{\nu}^{*} Y_{R} \\
& \beta_{Y_{R}}^{(2)}=\frac{1}{40}\left(-320 \lambda_{\sigma} Y_{R} Y_{R}^{*} Y_{R}+51 g_{1}^{2} Y_{\nu}^{T} Y_{\nu}^{*} Y_{R}+255 g_{2}^{2} Y_{\nu}^{T} Y_{\nu}^{*} Y_{R}-160 \lambda_{H \sigma} Y_{\nu}^{T} Y_{\nu}^{*} Y_{R}\right. \\
& -10 Y_{R} Y_{\nu}^{\dagger} Y_{\nu} Y_{\nu}^{\dagger} Y_{\nu}-10 Y_{R} Y_{\nu}^{\dagger} Y_{\nu} Y_{R}^{*} Y_{R}+70 Y_{R} Y_{R}^{*} Y_{R} Y_{R}^{*} Y_{R}-10 Y_{R} Y_{R}^{*} Y_{\nu}^{T} Y_{\nu}^{*} Y_{R} \\
& +160 Y_{\nu}^{T} Y_{\nu}^{*} Y_{R} Y_{\nu}^{\dagger} Y_{\nu}-10 Y_{\nu}^{T} Y_{\nu}^{*} Y_{\nu}^{T} Y_{\nu}^{*} Y_{R}-30 Y_{R} Y_{R}^{*} Y_{R} \operatorname{Tr}\left(Y_{R} Y_{R}^{*}\right)-180 Y_{\nu}^{T} Y_{\nu}^{*} Y_{R} y_{t}^{2} \\
& +Y_{R} Y_{\nu}^{\dagger} Y_{\nu}\left(-160 \lambda_{H \sigma}-180 y_{t}^{2}+255 g_{2}^{2}+51 g_{1}^{2}-60 \operatorname{Tr}\left(Y_{\nu} Y_{\nu}^{\dagger}\right)\right)-60 Y_{\nu}^{T} Y_{\nu}^{*} Y_{R} \operatorname{Tr}\left(Y_{\nu} Y_{\nu}^{\dagger}\right) \\
& \left.+10 Y_{R}\left(16 \lambda_{\sigma}^{2}-3 \operatorname{Tr}\left(Y_{R} Y_{R}^{*} Y_{R} Y_{R}^{*}\right)+4 \lambda_{H \sigma}^{2}-6 \operatorname{Tr}\left(Y_{R} Y_{\nu}^{\dagger} Y_{\nu} Y_{R}^{*}\right)\right)\right) \text {. }
\end{aligned}
$$

[1] G. Aad et al. (ATLAS Collaboration), Observation of a new particle in the search for the Standard Model Higgs boson with the ATLAS detector at the LHC, Phys. Lett. B 716, 1 (2012).

[2] S. Chatrchyan et al. (CMS Collaboration), Observation of a new boson at a mass of $125 \mathrm{GeV}$ with the CMS experiment at the LHC, Phys. Lett. B 716, 30 (2012).

[3] M. Tanabashi et al. (Particle Data Group), Review of particle physics, Phys. Rev. D 98, 030001 (2018).

[4] T. Kajita, Nobel Lecture: Discovery of atmospheric neutrino oscillations, Rev. Mod. Phys. 88, 030501 (2016).
[5] A. B. McDonald, Nobel Lecture: The Sudbury neutrino observatory: Observation of flavor change for solar neutrinos, Rev. Mod. Phys. 88, 030502 (2016).

[6] P. F. de Salas, D. V. Forero, C. A. Ternes, M. Tórtola, and J. W. F. Valle, Status of neutrino oscillations 2018: $3 \sigma$ hint for normal mass ordering and improved $C P$ sensitivity, Phys. Lett. B 782, 633 (2018).

[7] J. W. F. Valle and J. C. Romao, Neutrinos in high Energy and Astroparticle Physics (John Wiley \& Sons, New York, 2015).

[8] M. Lindner, H. H. Patel, and B. Radovčić, Electroweak absolute, meta-, and thermal stability in neutrino mass models, Phys. Rev. D 93, 073005 (2016). 
[9] G. Bambhaniya, P. Bhupal Dev, S. Goswami, S. Khan, and W. Rodejohann, Naturalness, vacuum stability and leptogenesis in the minimal Seesaw model, Phys. Rev. D 95, 095016 (2017).

[10] C. Bonilla, R. M. Fonseca, and J. W. F. Valle, Vacuum stability with spontaneous violation of lepton number, Phys. Lett. B 756, 345 (2016).

[11] L. Delle Rose, C. Marzo, and A. Urbano, On the stability of the electroweak vacuum in the presence of low-scale seesaw models, J. High Energy Phys. 12 (2015) 050.

[12] J. Schechter and J. W. F. Valle, Neutrino masses in $\mathrm{SU}(2) \times$ U(1) theories, Phys. Rev. D 22, 2227 (1980).

[13] N. Rojas, R. Srivastava, and J. W. Valle, Simplest ScotoSeesaw mechanism, Phys. Lett. B 789, 132 (2019).

[14] J. W. F. Valle, Neutrino physics outlook, 21st International Workshop on Neutrinos from Accelerators (Nufact19) Daegu, Korea (PoS NuFact2019, 2019).

[15] Y. Chikashige, R. N. Mohapatra, and R. D. Peccei, Are there real Goldstone Bosons associated with broken Lepton number?, Phys. Lett. 98B, 265 (1981).

[16] J. Schechter and J. W. F. Valle, Neutrino decay and spontaneous violation of Lepton number, Phys. Rev. D 25, 774 (1982).

[17] V. Berezinsky and J. W. F. Valle, The $\mathrm{KeV}$ majoron as a dark matter particle, Phys. Lett. B 318, 360 (1993).

[18] M. Lattanzi and J. W. F. Valle, Decaying Warm Dark Matter and Neutrino Masses, Phys. Rev. Lett. 99, 121301.

[19] F. Bazzocchi, M. Lattanzi, S. Riemer-Sørensen, and J. W. F. Valle, X-ray photons from late-decaying majoron dark matter, J. Cosmol. Astropart. Phys. 08 (2008) 013.

[20] M. Lattanzi, Signe Riemer-Sørensen, Mariam Tórtola, and J. W. F. Valle, Updated CMB, X-and gamma-ray constraints on Majoron dark matter, Phys. Rev. D 88, 063528 (2013).

[21] J.-L. Kuo, M. Lattanzi, K. Cheung, and J. W. F. Valle, Decaying warm dark matter and structure formation, J. Cosmol. Astropart. Phys. 12 (2018) 026.

[22] G. Isidori, G. Ridolfi, and A. Strumia, On the metastability of the standard model vacuum, Nucl. Phys. B609, 387 (2001).

[23] J. Elias-Miro, J. R. Espinosa, G. F. Giudice, G. Isidori, A. Riotto, and A. Strumia, Higgs mass implications on the stability of the electroweak vacuum, Phys. Lett. B 709, 222 (2012).

[24] F. Bezrukov, M. Y. Kalmykov, B. A. Kniehl, and M. Shaposhnikov, Higgs Boson mass and new physics, J. High Energy Phys. 10 (2012) 140.

[25] G. Degrassi, S. Di Vita, J. Elias-Miro, J. R. Espinosa, G. F. Giudice, G. Isidori, and A. Strumia, Higgs mass and vacuum stability in the Standard Model at NNLO, J. High Energy Phys. 08 (2012) 098.

[26] I. Masina, Higgs boson and top quark masses as tests of electroweak vacuum stability, Phys. Rev. D 87, 053001 (2013).

[27] D. Buttazzo, G. Degrassi, P. P. Giardino, G. F. Giudice, F. Sala, A. Salvio, and A. Strumia, Investigating the nearcriticality of the Higgs boson, J. High Energy Phys. 12 (2013) 089.

[28] S. Weinberg, Baryon and Lepton Nonconserving Processes, Phys. Rev. Lett. 43, 1566 (1979).
[29] R. N. Mohapatra and J. W. F. Valle, Neutrino mass and baryon-number nonconservation in superstring models, Phys. Rev. D 34, 1642 (1986).

[30] M. Gonzalez-Garcia and J. W. F. Valle, Fast decaying neutrinos and observable flavor violation in a new class of Majoron models, Phys. Lett. B 216, 360 (1989).

[31] E. K. Akhmedov, M. Lindner, E. Schnapka, and J. W. F. Valle, Left-right symmetry breaking in NJL approach, Phys. Lett. B 368, 270 (1996).

[32] E. K. Akhmedov, M. Lindner, E. Schnapka, and J. W. F. Valle, Dynamical left-right symmetry breaking, Phys. Rev. D 53, 2752 (1996).

[33] M. Malinsky, J. Romao, and J. W. F. Valle, Novel Supersymmetric SO(10) Seesaw Mechanism, Phys. Rev. Lett. 95, 161801 (2005).

[34] M. Aker et al. (KATRIN Collaboration), An Improved Upper Limit on the Neutrino Mass from a Direct Kinematic Method by KATRIN, Phys. Rev. Lett. 123, 221802 (2019).

[35] N. Aghanim et al. (Planck Collaboration), Planck 2018 results. VI. Cosmological parameters, arXiv:1807.06209.

[36] M. Lattanzi and M. Gerbino, Status of neutrino properties and future prospects-Cosmological and astrophysical constraints, Front. Phys. 5, 70 (2018).

[37] C. Alduino et al. (CUORE Collaboration), First Results from CUORE: A Search for Lepton Number Violation via $0 \nu \beta \beta$ Decay of ${ }^{130}$ Te, Phys. Rev. Lett. 120, 132501 (2018).

[38] J. Albert et al. (EXO Collaboration), Search for Neutrinoless Double-Beta Decay with the Upgraded EXO-200 Detector, Phys. Rev. Lett. 120, 072701 (2018).

[39] M. Agostini et al. (GERDA Collaboration), Improved Limit on Neutrinoless Double- $\beta$ Decay of ${ }^{76} \mathrm{Ge}$ from GERDA Phase II, Phys. Rev. Lett. 120, 132503 (2018).

[40] A. Gando et al. (KamLAND-Zen Collaboration), Search for Majorana Neutrinos near the Inverted Mass Hierarchy Region with KamLAND-Zen, Phys. Rev. Lett. 117, 082503 (2016).

[41] S. Andringa et al. (SNO+ Collaboration), Current Status and Future Prospects of the SNO + Experiment, Adv. High Energy Phys. 2016, 1 (2016).

[42] N. Abgrall et al. (LEGEND Collaboration), The large enriched Germanium experiment for neutrinoless double beta decay (LEGEND), AIP Conf. Proc. 1894, 020027 (2017).

[43] J. Albert et al. (nEXO Collaboration), Sensitivity and discovery potential of nEXO to neutrinoless double beta decay, Phys. Rev. C 97, 065503 (2018).

[44] P. H. Chankowski, A. N. Ioannisian, S. Pokorski, and J. W. F. Valle, Neutrino Unification, Phys. Rev. Lett. 86, 3488 (2001).

[45] S. Antusch, J. Kersten, M. Lindner, and M. Ratz, Neutrino mass matrix running for nondegenerate seesaw scales, Phys. Lett. B 538, 87 (2002).

[46] J. Bergstrom, T. Ohlsson, and H. Zhang, Threshold effects on renormalization group running of neutrino parameters in the low-scale seesaw model, Phys. Lett. B 698, 297 (2011).

[47] J. N. Ng and A. de la Puente, Electroweak vacuum stability and the Seesaw mechanism revisited, Eur. Phys. J. C 76, 122 (2016). 
[48] J. A. Casas, V. Di Clemente, A. Ibarra, and M. Quiros, Massive neutrinos and the Higgs mass window, Phys. Rev. D 62, 053005 (2000).

[49] I. Brivio and M. Trott, Radiatively Generating the Higgs Potential and Electroweak Scale via the Seesaw Mechanism, Phys. Rev. Lett. 119, 141801 (2017).

[50] P. Ghosh, A. K. Saha, and A. Sil, Study of electroweak vacuum stability from extended Higgs portal of dark matter and neutrinos, Phys. Rev. D 97, 075034 (2018).

[51] A. S. Joshipura and J. W. F. Valle, Invisible Higgs decays and neutrino physics, Nucl. Phys. B397, 105 (1993).

[52] J. Sirkka and I. Vilja, Vacuum stability in the singlet Majoron model, Phys. Lett. B 332, 141 (1994).

[53] J. Elias-Miro, J. R. Espinosa, G. F. Giudice, H. M. Lee, and A. Strumia, Stabilization of the electroweak vacuum by a scalar threshold effect, J. High Energy Phys. 06 (2012) 031.
[54] S. R. Coleman, Why there is nothing rather than something: A theory of the cosmological constant, Nucl. Phys. B310, 643 (1988).

[55] B. Audren, J. Lesgourgues, G. Mangano, P. D. Serpico, and T. Tram, Strongest model-independent bound on the lifetime of dark matter, J. Cosmol. Astropart. Phys. 12 (2014) 028.

[56] S. M. Boucenna, S. Morisi, Q. Shafi, and J. W. F. Valle, Inflation and majoron dark matter in the seesaw mechanism, Phys. Rev. D 90, 055023 (2014).

[57] A. Sierra, M. Tórtola, J. W. F. Valle, and A. Vicente, Leptogenesis with a dynamical seesaw scale, J. Cosmol. Astropart. Phys. 07 (2014) 052.

[58] F. Staub, Exploring new models in all detail with SARAH, Adv. High Energy Phys. 2015, 840780 (2015). 